\title{
Research on the Capability Maturity Evaluation of Intelligent Manufacturing Based on Firefly Algorithm, Sparrow Search Algorithm, and BP Neural Network
}

\author{
Li Shi $\mathbb{I D}^{1,2}$ Xuehong Ding $\mathbb{1 D}^{2}{ }^{2}$ Min $\mathrm{Li}^{2}$, and Yuan Liu ${ }^{3}$ \\ ${ }^{1}$ School of Management, Hefei University of Technology, Hefei 230009, China \\ ${ }^{2}$ School of Computer Science and Technology, Huaibei Normal University, Huaibei 235000, China \\ ${ }^{3}$ College of Economics and Management, Huaibei Normal University, Huaibei 235000, China
}

Correspondence should be addressed to Xuehong Ding; shili@chnu.edu.cn

Received 1 February 2021; Revised 5 July 2021; Accepted 30 July 2021; Published 21 August 2021

Academic Editor: Muhammad Ahmad

Copyright (c) $2021 \mathrm{Li}$ Shi et al. This is an open access article distributed under the Creative Commons Attribution License, which permits unrestricted use, distribution, and reproduction in any medium, provided the original work is properly cited.

\begin{abstract}
Intelligent manufacturing capability evaluation is the key for enterprises to scientifically formulate the implementation path and continuously improve the level of intelligent manufacturing. To help manufacturing enterprises diagnose the level of intelligent manufacturing capability, this paper conducts research on intelligent manufacturing capability maturity evaluation based on maturity theory. The evaluation problem is a complex nonlinear problem, and BP neural network is particularly suitable for solving such complex mapping problems. Aiming at the problem that the BP neural network is sensitive to initial weights and thresholds, the sparrow search algorithm (SSA) is used to optimize the initial weights and thresholds of the BP neural network. In order to overcome the shortcoming of SSA that it is easy to fall into the local optimum, the firefly disturbance strategy is introduced to improve it, a new sparrow search algorithm (FASSA) is proposed, and on this basis, an intelligent manufacturing capability maturity evaluation model based on the FASSA-BP algorithm is constructed. Finally, a large battery manufacturing enterprise in China is selected for empirical research, and the comparison experiments are carried out on the FASSA-BP model, BP model, SSA-BP model, and PSO-BP model in terms of accuracy, stability, etc. The results show that the evaluation of intelligent manufacturing capability maturity through this model can effectively help companies diagnose problems in the construction of intelligent manufacturing and provide a reference for companies to accurately improve their intelligent manufacturing capabilities.
\end{abstract}

\section{Introduction}

With the deep integration of information technology, intelligent technology, and manufacturing technology, intelligent manufacturing has received great attention from governments and industries [1-3]. As the core of the new round of industrial revolution, intelligent manufacturing is leading the intelligent transformation of manufacturing enterprises globally, and the evaluation of intelligent manufacturing capability has become the key for enterprises to scientifically formulating the implementation path and continuously improving the level of intelligent manufacturing. In the implementation and promotion of intelligent manufacturing, many enterprises do not know enough about their overall intelligent manufacturing development level and lack effective methods to diagnose intelligent manufacturing capability and identify gaps. How to carry out evaluation for enterprise intelligent manufacturing capability has become a new research area of concern for scholars at home and abroad, with the study of intelligent manufacturing capability evaluation based on maturity theory having become an important research direction.

The concept of intelligent manufacturing capability maturity (IMCM) is not yet uniformly defined. Different organizations and institutions have given their 
understanding from different perspectives. De Carolis et al. evaluate the key processes of intelligent manufacturing from five different dimensions and, inspired by the integration framework of capability maturity models, propose an intelligent manufacturing capability maturity model [4]. Based on the "Made in China 2025" plan and combined with capability maturity model theory, the intelligent manufacturing maturity model was studied by $\mathrm{Hu}$ and Gao based on China's national conditions from three aspects: maturity level, capability factor, and maturity requirements [5]. Zhou et al. studied the issues related to the transformation and upgrading of intelligent manufacturing in Chinese enterprises and found that Chinese manufacturing enterprises were able to develop their own intelligent manufacturing capability upgrading paths based on their own capabilities and industry characteristics [6]. From the perspective of smart factories, Ren et al. firstly proposed the capability maturity model of China's petrochemical industry [7]. Simetinger and Zhang conducted a comparative analysis of several important maturity models to identify common features of Industry 4.0 maturity models [8].

The maturity of intelligent manufacturing capability can reflect the development level of intelligent manufacturing of enterprises, but it also needs to be evaluated with specific evaluation methods. Researchers from different countries have explored the problem of evaluating the maturity of intelligent manufacturing capabilities and the construction of evaluation models.

Wagire et al. used fuzzy hierarchical analysis to determine an Industry 4.0 maturity assessment model and implemented an evaluation of an automotive parts manufacturing company based on this, and the study showed that the model is feasible in practical application and easy to self-evaluate [9]. Ruiz et al. established a capability maturity evaluation model by fuzzy integrated evaluation method to clarify the level of enterprise intelligent manufacturing [10]. Colli et al. proposed a new 360 digit maturity assessment method based on the learning theory model, and the results of the study showed that the method can provide actionable recommendations for enterprises, making the results of Industry 4.0 capability maturity evaluation concrete [11]. Lee et al. constructed a smart factory assessment framework based on the concept of operations management, used network analysis to analyze and evaluate it, and verified its effectiveness through case studies [12]. Liu et al. explored the research issue about carbon market maturity evaluation in depth and proposed the entropy-based TOPSIS model to measure the maturity level of carbon market [13]. To determine the development level of intelligent manufacturing maturity, Xiao et al. combined hierarchical analysis with Hopfield neural network and conducted an empirical study based on it, so as to clarify the usability of the proposed method [14]. Xu and Xiao used the fuzzy comprehensive evaluation method to carry out the study of intelligent manufacturing capability [15]. Meanwhile, Zhi-qiang used the mutation level method in order to achieve the metric of intelligent manufacturing capability [16]. Shao and Wen used factor analysis method as an evaluation method of intelligent manufacturing capability and used some Chinese provinces as a research sample to obtain the capability ranking of each province after evaluation [17].

The above-mentioned scholars have conducted research on the maturity of intelligent manufacturing capability from different perspectives, providing a certain theoretical basis and methodological reference for the evaluation of intelligent manufacturing capability maturity. Although certain research results have been achieved, the overall research is still in the initial stage of exploration; in particular, in the evaluation method, there is still room for improvement. The concrete performance is that the existing research mostly adopts traditional evaluation methods such as analytic hierarchy process (AHP) and factor analysis. Although the original information of most indicators is retained, the evaluation process is obviously subjective due to the influence of expert experience in determining the weight. In addition, these methods still have some defects, such as heavy workload, complex calculation, and being timeconsuming.

In order to overcome the shortage of traditional evaluation methods of capability maturity, this paper applies intelligent algorithms in the field of computer science; on the basis of constructing evaluation indexes of enterprise intelligent manufacturing capability maturity, uses intelligent methods such as sparrow search algorithm (SSA), firefly algorithm (FA), and BP (back propagation) neural network; proposes an intelligent manufacturing capability maturity evaluation model based on FASSA-BP algorithm; and applies it to a large battery manufacturing enterprise in China for IMCM evaluation research. Through comparative experiments, the validity and applicability of the evaluation model are verified.

\section{Constructing the IMCM Evaluation Index}

Combining intelligent manufacturing capability and existing maturity theory and referring to the documents related to intelligent manufacturing capability maturity released at home and abroad $[2,3]$, this paper considers that intelligent manufacturing capability maturity is a comprehensive measure of the whole process of intelligent manufacturing in enterprises and describes the evolution path of intelligent manufacturing from unplanned level to leading level.

Based on the above-mentioned understanding of the maturity of intelligent manufacturing capabilities, following the construction principles of the indicator system $[18,19]$, and according to the industry characteristics of intelligent manufacturing enterprises, we construct the evaluation index system of intelligent manufacturing capabilities, providing methods for enterprises to carry out self-diagnosis of intelligent manufacturing capabilities. The system includes one first-level index of strategy and organization, design, production, equipment, warehouse, sales, service, network environment, network security, and architecture platform and refines 20 second level indexes to obtain the index system shown in Figure 1. 


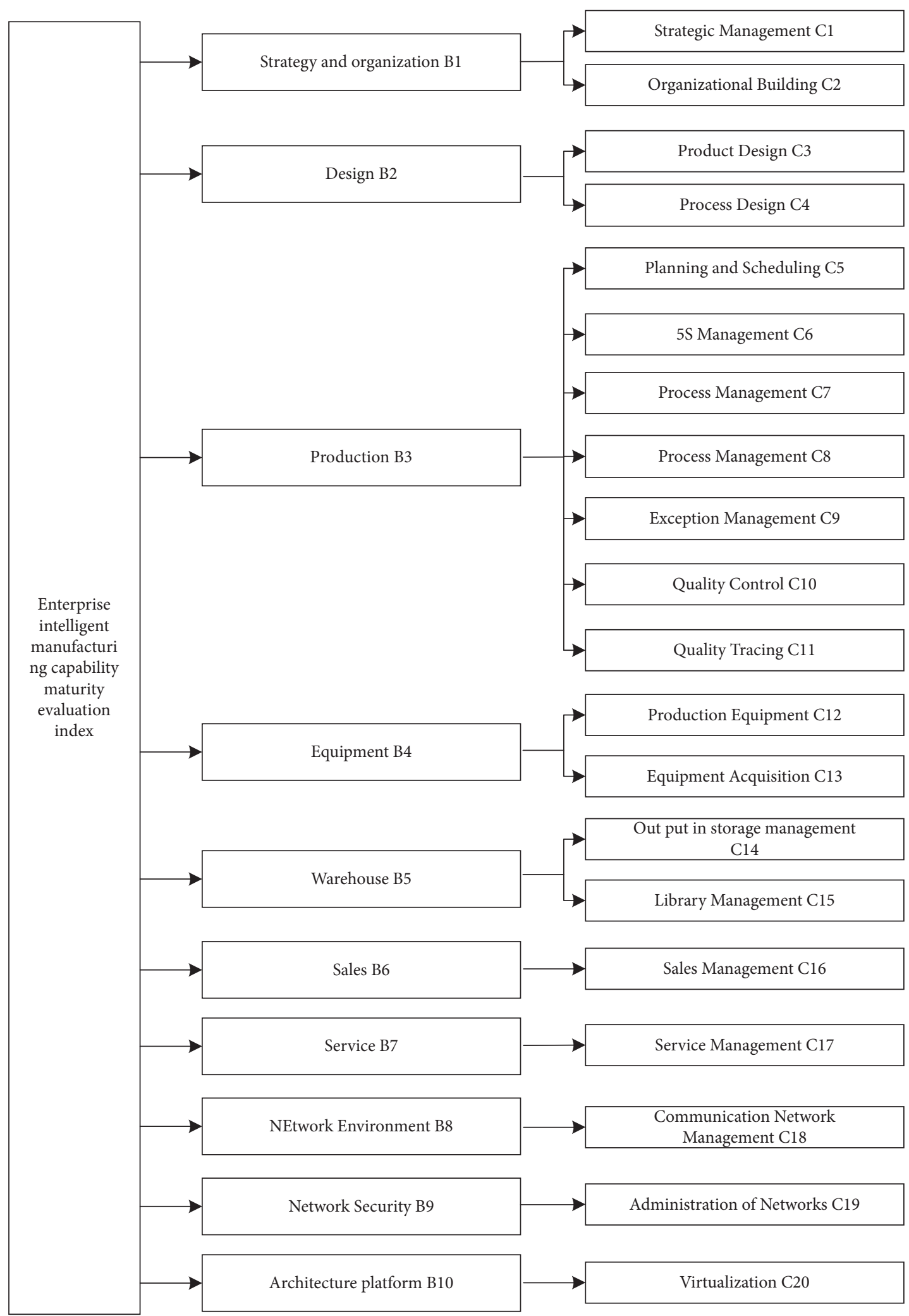

Figure 1: Intelligent manufacturing capability evaluation index system.

Through establishing the evaluation index system, the evaluation of the maturity of the enterprise's intelligent manufacturing capability can be based on the evidence. We use the evaluation index system to design questionnaires to collect sample data. Firstly, the questionnaire questions are designed according to the 20 secondary indicators that have 
been constructed; that is, each secondary indicator corresponds to one question. There are 20 multiple-choice questions in total; each question has six options of ABCDEF, and each option corresponds to a score of $0,20,40,60,80$, 100. The collected sample data is the score of each question in the questionnaire, that is, the score of each secondary evaluation index. Therefore, after each questionnaire is filled out by each person, the selected option is converted into the corresponding score to get the sample data.

\section{Improving the Sparrow Search Algorithm}

3.1. Basic Sparrow Search Algorithm. The sparrow search algorithm is an intelligent optimization algorithm based on the foraging and antipredation behaviors of sparrow populations proposed by Xue and Shen in 2020 [20]. It has three behavioral strategies: producer searching for food [21], follower acquiring food through producer [22], and antipredation [23]. Among them, the producer is responsible for finding food and directing the flow of the whole population. Once an individual finds a predator, it signals an alarm, and when the alarm value is greater than the safety threshold, the producer directs all followers to a safe area. During each iteration, the producer's position is updated as follows:

$$
X_{i, j}^{t+1}=\left\{\begin{array}{l}
X_{i, j}^{t} \cdot \exp \left(\frac{-i}{\alpha \cdot \text { iter }_{\max }}()_{2}\right) \\
X_{i, j}^{t}+\text { Q.Lif } R_{2} \geq S T
\end{array}\right.
$$

In the formula, $t$ represents the current iteration number, $j=1,2, \ldots, d$. $X_{i, j}^{t}$ represents the value of the $j$ th dimension of the ith sparrow when the number of iterations is $t$. iter ${ }_{\max }$ is the constant with the maximum number of iterations. $\alpha \in(0,1]$ is a random number. $R_{2}\left(R_{2} \in[0,1]\right)$ and $S T(S T \in[0.5,1.0])$ denote the alarm value and safety threshold. $Q$ is a random number obeying normal distribution. $L$ denotes a $1 \times d$ matrix, where each element is 1 . When $R_{2}<S T$, this means that there are no predators around the population and the producer enters the widearea search mode. If $R_{2} \geq S T$, this means that some sparrows have found predators and all sparrows need to fly quickly to other safe areas.

For followers, sparrows that are in a very hungry position forage elsewhere, while some followers constantly monitor producers and compete for food to increase their own predation rate. The updated formula of follower's position is as follows:

$$
X_{i, j}^{t+1}= \begin{cases}Q \cdot \exp \left(\frac{x_{\mathrm{worst}}^{t}-x_{i, j}^{t}}{i^{2}}\right), & \text { if } i>\frac{n}{2}, \\ X_{p}^{t+1}+\left|X_{i, j}^{t}-X_{p}^{t+1}\right| \cdot A^{+} \cdot L, & \text { otherwise }\end{cases}
$$

where $X_{p}$ is the best position occupied by the producer. $X_{\text {worst }}$ denotes the current global worst position. A denotes a $1 \times d$ matrix where each element is randomly assigned as 1 or $-1, A^{+}=A^{T}\left(A A^{T}\right)^{-1}$. When $i>n / 2$ indicates that the ith follower with the worse fitness value is most likely to be in hunger.

Sparrows that are aware of the danger represent 10-20\% of the total population. The initial positions of these sparrows are generated randomly in the population. The updated formula of these sparrows' position is as follows:

$$
X_{i, j}^{t+1}= \begin{cases}X_{\text {best }}^{t}+\beta \cdot\left|X_{i, j}^{t}-X_{\text {best }}^{t}\right| & \text { if } f_{i}>f_{g}, \\ X_{i, j}^{t}+K \cdot\left(\frac{\left|X_{i, j}^{t}-X_{\text {worst }}^{t}\right|}{\left(f_{i}-f_{w}\right)+\varepsilon}\right) & \text { if } f_{i}=f_{g},\end{cases}
$$

where $X_{\text {best }}$ is the current global optimal position. As the step control parameter, $\beta$ is a normally distributed random number with mean 0 and variance $1 . K \in[-1,1]$ is a random number. $f_{i}$ is the current adaptation value of the sparrow. $f_{g}$ and $f_{w}$ are, respectively, the current global best and worst fitness values. $\varepsilon$ is the minimum constant. When $f_{i}>f_{g}$, this indicates that the sparrow is located at the edge of the population. $X_{\text {best }}$ represents the position in the center of the population and the surroundings are safe. $f_{i}=f_{g}$ indicates that the sparrow in the middle of the population is aware of the danger and needs to move closer to other sparrows. $K$ indicates the direction of sparrow movement and it is also the step control coefficient.

The SSA has a strong partial search capability, but it still needs to be enhanced in terms of global search and jumping out of local optimum. Thus, it affects its convergence accuracy, but this deficiency belongs to the common problem of most optimization algorithms [24]. At present, the SSA is still a newly developed algorithm, and there are still relatively few related papers and studies, so there is still some room for improvement in the current SSA.

3.2. Improving SSA Based on FA. Aiming at remedying the shortcomings of the above-mentioned SSA, we introduce the firefly algorithm (FA), and the SSA search efficiency is improved mainly through the firefly disturbance strategy. The improved SSA can continue to search when the local extreme value is reached to obtain the optimal solution.

3.2.1. Firefly Perturbation Strategy. In the firefly algorithm $[25,26]$, the fireflies rely mainly on their own brightness to communicate information. Each firefly has brightness and attractiveness, and the brightness is used to determine the current position in order to choose its next flight direction, while the firefly's position update in space is influenced by attractiveness. These two are positively related, and fireflies will be attracted by ones brighter than them in order to constantly seek the best.

(1) The attractiveness of fireflies is

$$
\beta=\beta_{0} * e^{-\gamma r_{i, j}^{2}}
$$

where $\beta_{0}$ is its initial attraction; $\gamma$ is the light intensity absorption coefficient; and $r_{i . j}$ represents the distance between the fireflies $i$ and $j$. 
(2) When the firefly $i$ is brighter than $j, i$ update the position according to the following formula:

$$
x_{i}=x_{i}+\beta *\left(x_{j}-x_{i}\right)+\alpha *\left(\operatorname{rand}-\frac{1}{2}\right),
$$

where $x_{j}, x_{i}$ are, respectively, the position of fireflies $i$ and $j . \alpha$ denotes the disturbed step size factor, $\alpha \in[0,1]$. rand is a random perturbation, uniformly distributed, and takes values in the range $[0,1]$.

\subsubsection{Improving SSA Using the Firefly Perturbation Strategy.} In this paper, the idea of improving the SSA is as follows: after the sparrow search, using the firefly perturbation strategy to perturb the sparrow, using the firefly disturbance method for all sparrows and the best sparrows to update their positions, improving its searchable and comparing the sparrows after the disturbance with the sparrows before the perturbation; if better, then the sparrows' position is updated; the improved SSA is called FASSA. The procedure of FASSA is as follows:

Step 1: initialize the population, the number of iterations, and the proportion of discoverers and joiners

Step 2: calculate the fitness values and rank them

Step 3: sparrow updates the finders' location according to formula (1)

Step 4: sparrow updates the followers' position according to formula (2)

Step 5: select the vigilantes randomly and update their positions according to formula (3)

Step 6: calculate the fitness value and update the sparrows' position

Step 7: use the firefly perturbation strategy; update the sparrows' positions according to formula (5)

Step 8: calculate the fitness value and update the sparrows' position

Step 9: if the end requirement is reached, output the result. If not, continuously execute Steps $2-8$

3.2.3. FASSA Validation and Comparison. In order to verify the feasibility and effectiveness of the FASSA proposed in this paper, we select high-dimensional unimodal, high-dimensional multimodal, and low-dimensional functions from the benchmark test function set as the test functions for verification and comparison in this paper, as shown in Table 1, and compare the test results with the basic SSA.

The experimental environment is as follows: Intel $^{\oplus}$ Core $^{\mathrm{TM}}$ i5-4210U processor, 4GB memory capacity, and Windows 10 64-bit operating system, with Matlab 2017b as the experimental software tool.

The number of populations and the maximum number of iterations are set to 30 and 1000, respectively. The dimension and search space settings of the test function are shown in Tables 1; within the maximum number of iterations, the optimal value and convergence speed of the test function finally found are selected as the evaluation index for comparing the performance of the FASSA and the SSA. The experimental results of the two algorithms for the 13 test functions are shown in Figures 2-14.

By analyzing the performance of the two algorithms on the above multiple functions, it can be seen that the FASSA proposed in this paper shows good convergence and search ability compared to the basic SSA on the highdimensional unimodal test functions. On the one hand, for the high-dimensional unimodal test functions, the performance of FASSA on $F_{1}-F_{3}$ test functions is obviously better, and for $F_{4}$ and $F_{5}$, in the case of falling into local extremums, FASSA can jump out in time and continue to search to find the optimal value. On the other hand, for the high-dimensional multimodal and low-dimensional test functions, FASSA also deals with relatively better results. As can be seen from the results, on functions $F_{7}, F_{8}$, and $F_{12}$, the FASSA gets good fitness values at the beginning of the iteration, while it has absolute advantages in $F_{6}, F_{9}, F_{10}, F_{11}$, and $F_{13}$ test functions, being obviously better than the basic SSA.

In summary, compared with the SSA, the FASSA proposed in this paper not only improves the convergence speed, but also has a higher accuracy in finding the best performance. Therefore, the improved FASSA is applied to optimize the BP neural network to build an intelligent manufacturing capability maturity evaluation model in this paper.

\section{Evaluating IMCM Based on FASSA-BP Algorithm}

4.1. BP Neural Network. Considering that the research object of this paper is the evaluation of enterprise intelligent manufacturing capability maturity, the problem studied is complex and nonlinear. However, BP neural network can realize mapping function from input to output, which is especially suitable for solving complex mapping problems with internal mechanism [27]. It can also self-adapt and learn. It can not only quantitatively analyze problems, but also expand the knowledge learned by applying network models [28]. Therefore, selecting BP neural network algorithm as the evaluation method of this paper can effectively avoid the interference of human subjective consciousness in the evaluation process.

BP neural network is a multilayer feed-forward neural network with error back propagation [29]. It consists of input layer, hidden layer, and output layer. Each layer has a large number of different neurons [30]. In the learning process of BP neural network, it is mainly composed of forward propagation of signal and backward propagation of error [31]. Forward propagation means that the input data are transmitted layer by layer to each hidden layer through the input layer and finally to the output layer. The back propagation of error is that if there is an error between the training output and the expected output in the forward propagation process, the error signal is 
TABLE 1: Test functions.

\begin{tabular}{|c|c|c|c|c|}
\hline Type & Baseline test functions & Dimensionality & $\begin{array}{c}\text { Search } \\
\text { space }\end{array}$ & $\begin{array}{l}\text { Optimum } \\
\text { value }\end{array}$ \\
\hline $\begin{array}{l}\text { High-dimensional } \\
\text { unimodal }\end{array}$ & $F_{1}(x)=\sum_{i=1}^{n} x_{i}^{2}$ & 30 & {$[-100,100]$} & 0 \\
\hline $\begin{array}{l}\text { High-dimensional } \\
\text { unimodal }\end{array}$ & $F_{2}(x)=\sum_{i=1}^{n}\left|x_{i}\right|+\prod_{i=1}^{n}\left|x_{i}\right|$ & 30 & {$[-10,10]$} & 0 \\
\hline $\begin{array}{l}\text { High-dimensional } \\
\text { unimodal }\end{array}$ & $F_{3}(x)=\sum_{i=1}^{n}\left(\sum_{j=1}^{i} x_{j}\right)^{2}$ & 30 & {$[-100,100]$} & 0 \\
\hline $\begin{array}{l}\text { High-dimensional } \\
\text { unimodal }\end{array}$ & $F_{4}(x)=\sum_{i=1}^{n-1}\left[100\left(x_{i+1}-x_{i}^{2}\right)^{2}+\left(x_{i}-1\right)^{2}\right]$ & 30 & {$[-30,30]$} & 0 \\
\hline $\begin{array}{l}\text { High-dimensional } \\
\text { unimodal }\end{array}$ & $F_{5}(x)=\sum_{i=1}^{n} i x_{i}^{4}+\operatorname{random}[0,1)$ & 30 & {$[-1.28,1.28]$} & 0 \\
\hline $\begin{array}{l}\text { High-dimensional } \\
\text { multimodal }\end{array}$ & $F_{6}(x)=\sum_{i=1}^{n}-x_{i} \sin \left(\sqrt{\left|x_{i}\right|}\right)$ & 30 & {$[-500,500]$} & $-418.9829 n$ \\
\hline $\begin{array}{l}\text { High-dimensional } \\
\text { multimodal }\end{array}$ & $\begin{aligned} F_{7}(x)=-20 \exp ( & \left.-0.2 \sqrt{(1 / n) \sum_{i=1}^{n} x_{i}^{2}}\right)- \\
& \exp \left(1 / n \sum_{i=1}^{n} \cos \left(2 \pi x_{i}\right)\right)+20+e\end{aligned}$ & 30 & {$[-32,32]$} & 0 \\
\hline $\begin{array}{l}\text { High-dimensional } \\
\text { multimodal }\end{array}$ & $F_{8}(x)=1 / 4000 \sum_{i=1}^{n} x_{i}^{2}-\prod_{i=1}^{n} \cos \left(x_{i} / \sqrt{i}\right)+1$ & 30 & {$[-600,600]$} & 0 \\
\hline $\begin{array}{l}\text { High-dimensional } \\
\text { multimodal }\end{array}$ & $F_{9}(x)=\pi / n\left\{\begin{array}{l}10 \sin \left(\pi y_{1}\right)+ \\
\sum_{i=1}^{n-1}\left(y_{i}-1\right)^{2}\left[1+10 \sin ^{2}\left(\pi y_{i+1}\right)\right]+\left(y_{n}-1\right)^{2}\end{array}\right.$ & 30 & {$[-50,50]$} & 0 \\
\hline $\begin{array}{l}\text { Low-dimensional } \\
\text { function }\end{array}$ & $F_{10}(x)=4 x_{1}^{2}-2.1 x_{1}^{4}+1 / 3 x_{1}^{6}+x_{1} x_{2}-4 x_{2}^{2}+4 x_{2}^{4}$ & 2 & {$[-5,5]$} & -1.0316 \\
\hline $\begin{array}{l}\text { Low-dimensional } \\
\text { function }\end{array}$ & $\begin{array}{c}F_{11}(x)=\left[1-\left|\sin \left[\pi\left(x_{1}-2\right)\right] \sin \left[\pi\left(x_{2}-2\right)\right] / \pi^{2}\left(x_{1}-2\right)\left(x_{2}-2\right)\right|^{5}\right] \\
{\left[2+\left(x_{1}-7\right)^{2}+2\left(x_{2}-7\right)^{2}\right]}\end{array}$ & 2 & {$[0,14]$} & 0 \\
\hline $\begin{array}{l}\text { Low-dimensional } \\
\text { function }\end{array}$ & $F_{12}(x)=\sum_{i=1}^{11}\left(a_{i}-\left(x_{1}\left(b_{i}^{2}+b_{i} x_{2}\right) / b_{i}^{2}+b_{i} x_{3}+x_{4}\right)\right)^{2}$ & 4 & {$[-5,5]$} & 0.000307 \\
\hline $\begin{array}{l}\text { Low-dimensional } \\
\text { function }\end{array}$ & $F_{13}(x)=-\sum_{i=1}^{4} c_{i} \exp \left(-\sum_{j=1}^{6} a_{i j}\left(x_{j}-p_{i j}\right)^{2}\right)$ & 6 & {$[0,1]$} & -3.32 \\
\hline
\end{tabular}

TABLE 2: Expected output value of the sample data.

\begin{tabular}{lcccccccc}
\hline Sample & $E$ & Order of evaluation & Sample & $E$ & Order of evaluation & Sample & $E$ & Order of evaluation \\
\hline$S_{1}$ & 68 & Level three & $\ldots$ & $\ldots$ & $\ldots$ & $S_{491}$ & 55 & Level three \\
$S_{2}$ & 49 & Level two & $\ldots$ & $\ldots$ & $\ldots$ & $S_{492}$ & 69 & Level three \\
$S_{3}$ & 41 & Level two & $S_{487}$ & 44 & Level two & $S_{493}$ & 53 & Level three \\
$S_{4}$ & 49 & Level two & $S_{488}$ & 43 & Level two & $S_{494}$ & 64 & Level three \\
$S_{5}$ & 57 & Level three & $S_{489}$ & 29 & Level one & $S_{495}$ & 62 & Level three \\
$\ldots$ & $\ldots$ & $\ldots$ & $S_{490}$ & 54 & Level three & $S_{496}$ & 72 & Level four \\
\hline
\end{tabular}

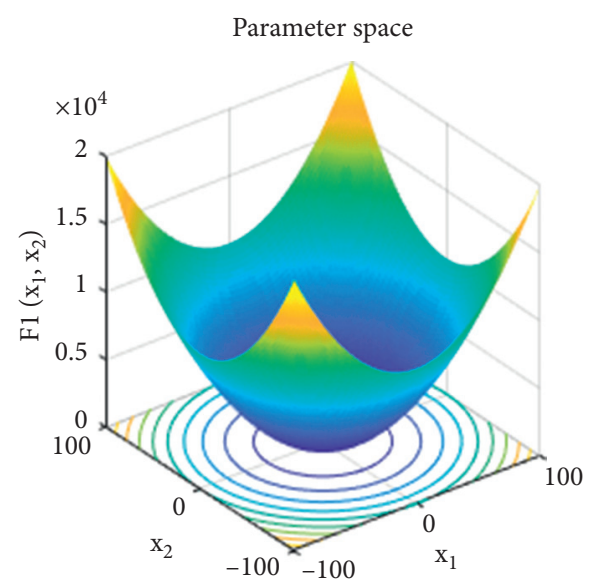

(a)

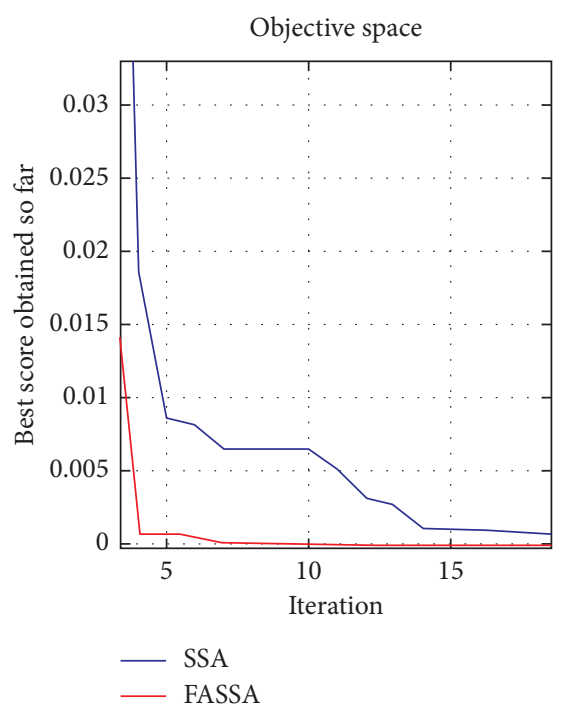

(b)

FIgURE 2: Experimental results of the test function $F_{1}$. 

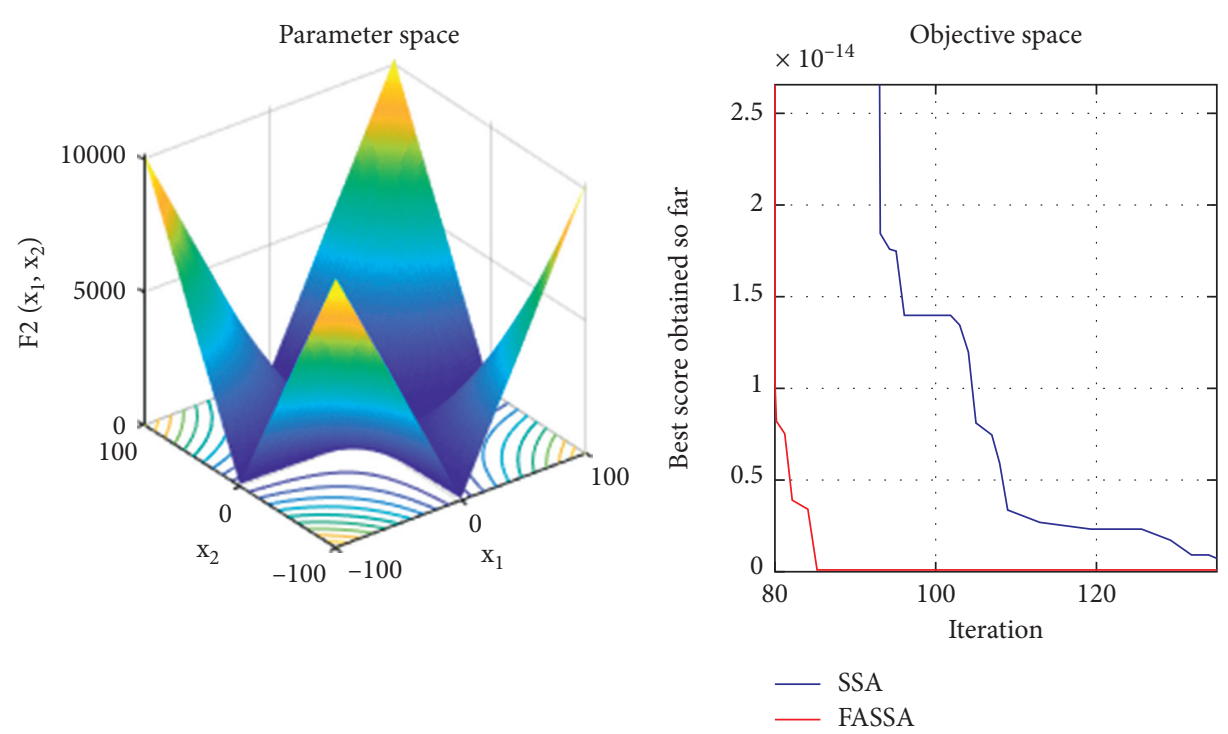

(a)

(b)

Figure 3: Experimental results of the test function $F_{2}$.

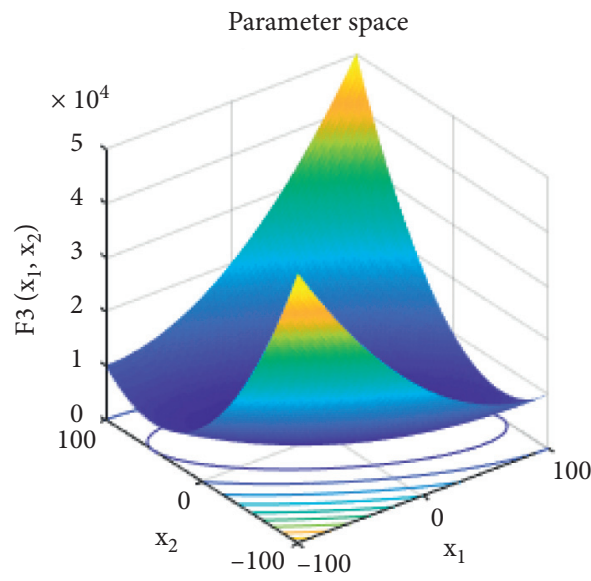

(a)

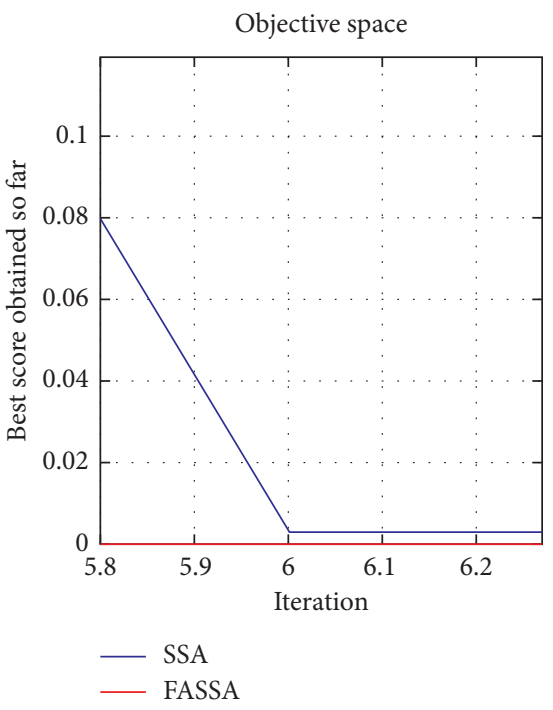

(b)

Figure 4: Experimental results of the test function $F_{3}$.

transmitted back to the hidden layer and the input layer by the gradient descent method, and the weights and thresholds of each neuron are continuously adjusted until the output error of BP neural network reaches the set accuracy standard or meets the number of iterations. The structure of the BP neural network is shown in Figure 15.

4.2. Optimizing the BP Neural Network Based on FASSA. In view of the shortcomings of BP neural network, that it is, being easy to fall into local optimum and randomly initializing weights and thresholds [32], FASSA is used to optimize the initial weights and thresholds of BP neural network, so as to overcome the shortcomings of $\mathrm{BP}$ neural network and improve the accuracy of intelligent manufacturing capability maturity evaluation model. The specific optimization process is shown in Figure 16.

The steps of optimizing BP neural network based on FASSA are as follows:

Step 1: design the structure of the BP neural network firstly.

Step 2: initialize FASSA population. Calculate the search space.

Dimension of FASSA based on the BP neural network topology structure. The calculating formula is described as follows: 

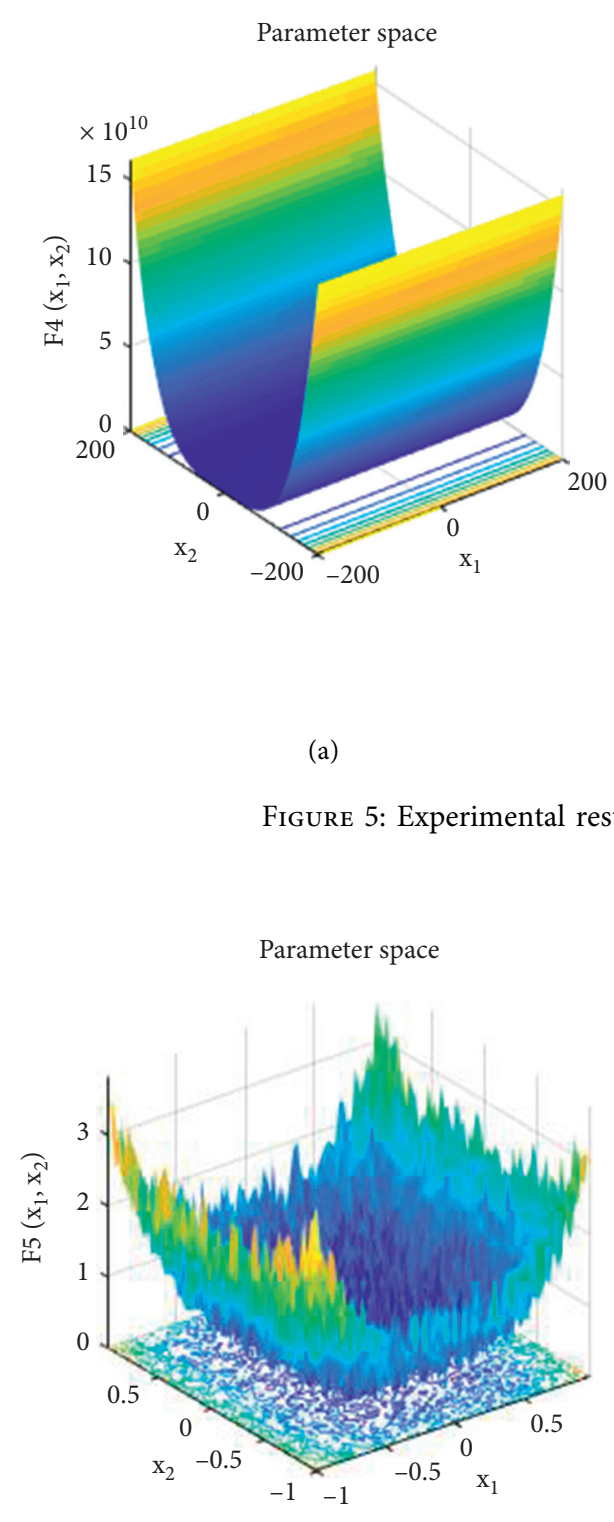

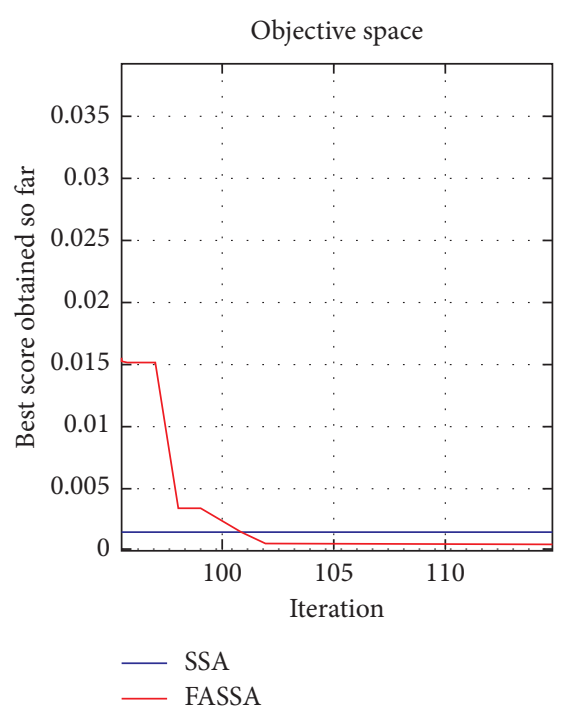

(b)

FIGURE 5: Experimental results of the test function $F_{4}$.

(a)

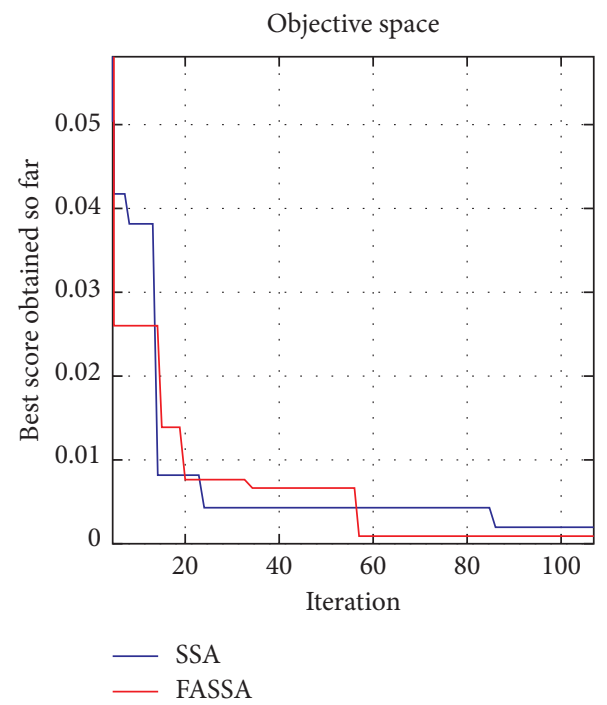

(b)

Figure 6: Experimental results of the test function $F_{5}$.

$$
\begin{aligned}
\text { Dim }= & \text { inputnum } * \text { hiddennum } \\
& + \text { outputnum } * \text { hiddennum } \\
& + \text { hiddennum }+ \text { outputnum }
\end{aligned}
$$

In the calculating formula, inputnum, outputnum, and hiddennum are the number of nodes in the input layer, output layer, and hidden layer.

Step 3: for the objective function of FASSA, the network error function is selected to design it:

$$
\text { Fitness }=\frac{1}{n} \sum_{i}^{n}\left(\widehat{y}_{i}-y_{i}\right)^{2} \text {. }
$$

In the formula, $n$ is the number of samples, $y_{i}$ represents the expected output value of the sample, and $\hat{y}_{i}$ represents the model output value of the sample.

Step 4: the position of sparrows is updated iteratively according to the formula for updating the position of sparrows with different roles in the basic SSA and calculating the fitness value. 

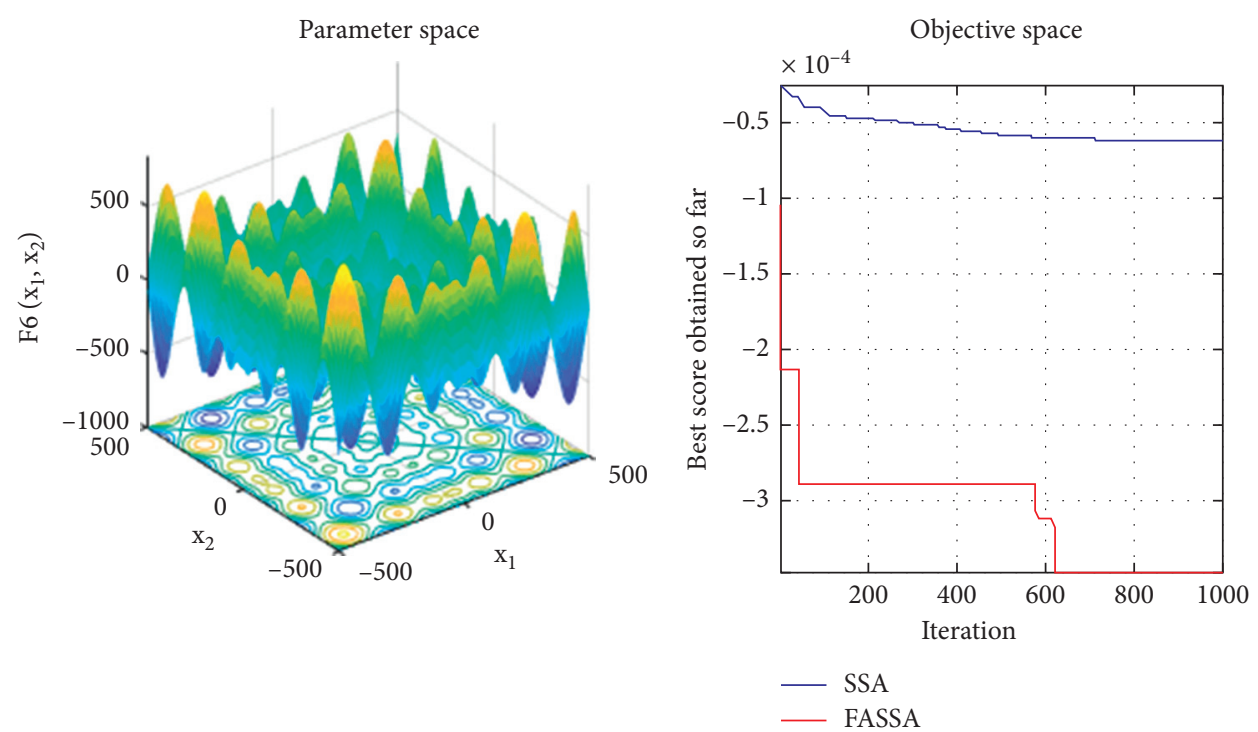

(a)

(b)

Figure 7: Experimental results of the test function $F_{6}$.
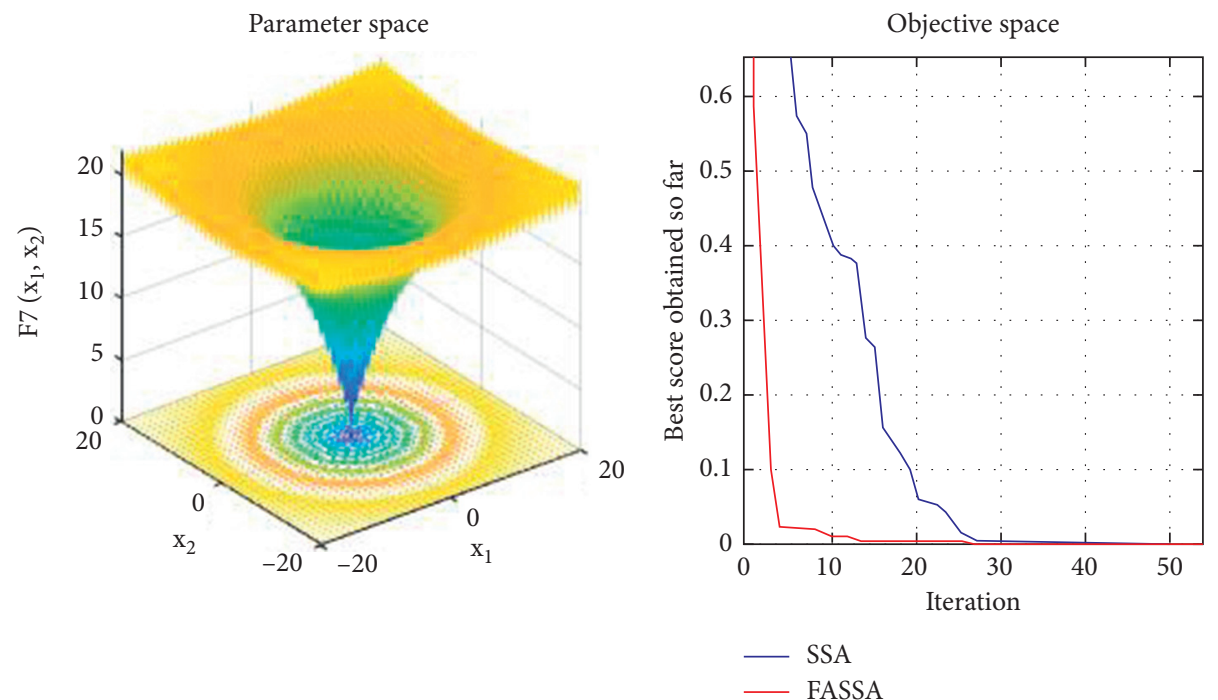

(a)

(b)

Figure 8: Experimental results of the test function $F_{7}$.

Step 5: use the firefly disturbance strategy to update and iteratively optimize the sparrows' position until the optimal solution is found or the end condition is satisfied.

Step 6: the FASSA is stopped to search, and the final solution is divided into the initial weights and thresholds between different neurons in BP neural network; that is, the optimal solution matrix vector obtained from FASSA is assigned. Among them, the weight matrix from the initial input layer to the hidden layer is

$$
W_{1}=x(1: \text { inputnum } * \text { hiddennum }) .
$$

The initial hidden layer threshold matrix is

$$
\begin{aligned}
B_{1}= & x \text { (inputnum } * \text { hiddennum }+1: \text { inputnum } \\
& * \text { hiddennum }+ \text { hiddennum }) .
\end{aligned}
$$

The weight matrix from the initial hidden layer to the output layer is 

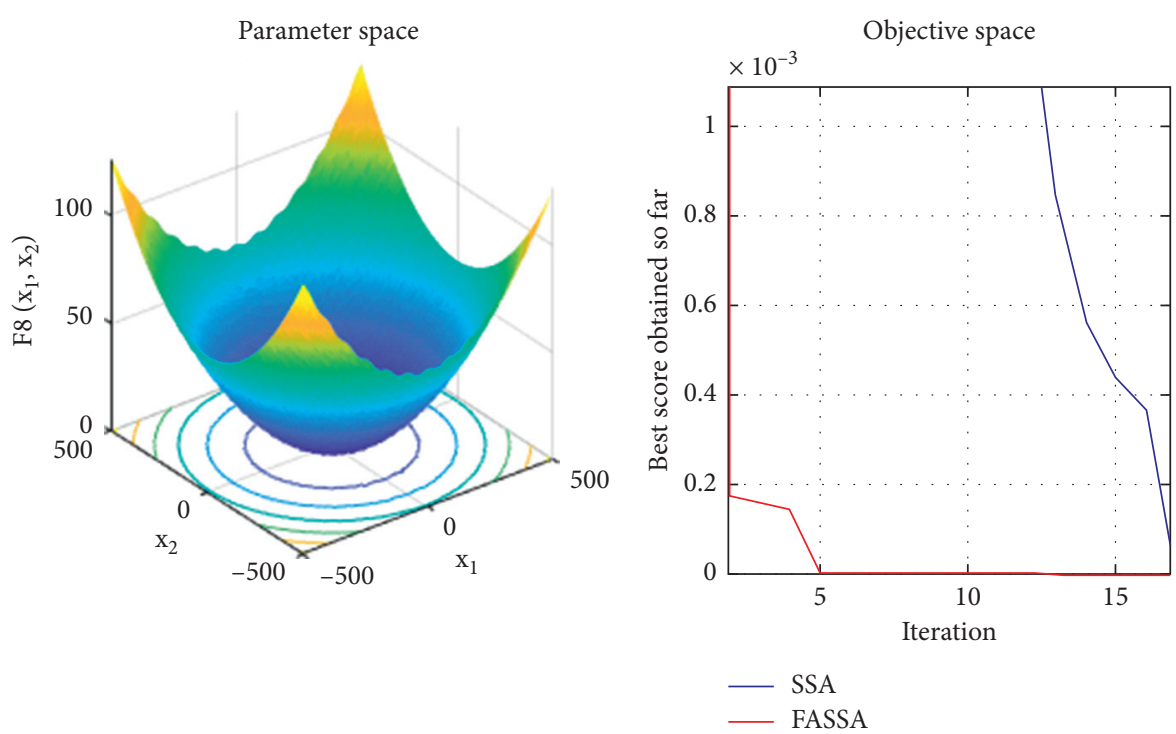

(a)

(b)

FIGURE 9: Experimental results of the test function $F_{8}$.
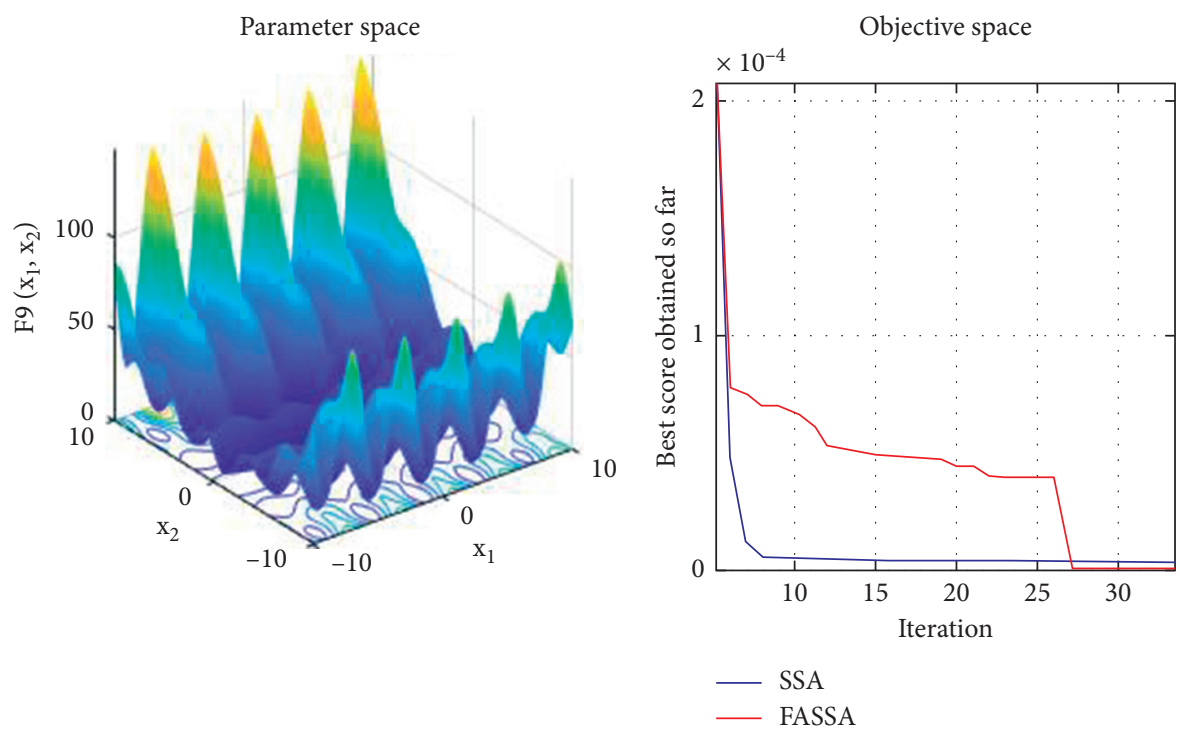

(a)

(b)

FIGURE 10: Experimental results of the test function $F_{9}$.

$W_{2}=x($ inputnum $*$ hiddennum + hiddennum $+1:$ inputnum $*$ hiddennum + hiddennum + hiddennum $*$ outputnum $)$,

and the threshold matrix of the output layer is

$B_{2}=x$ (inputnum $*$ hiddennum + hiddennum + hiddennum $*$ outputnum $+1:$ inputnum $*$ hiddennum + hiddennum + hiddennum $*$ outputnum + outputnum). 

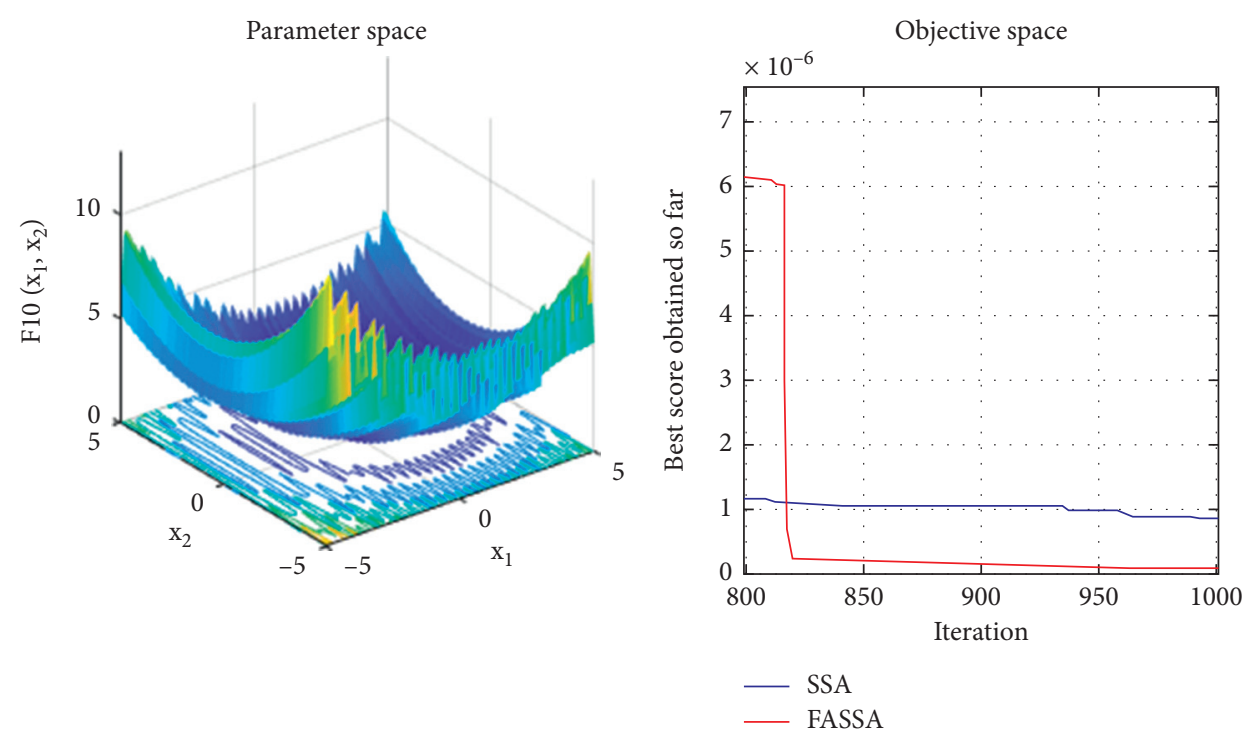

(a)

(b)

Figure 11: Experimental results of the test function $F_{10}$.
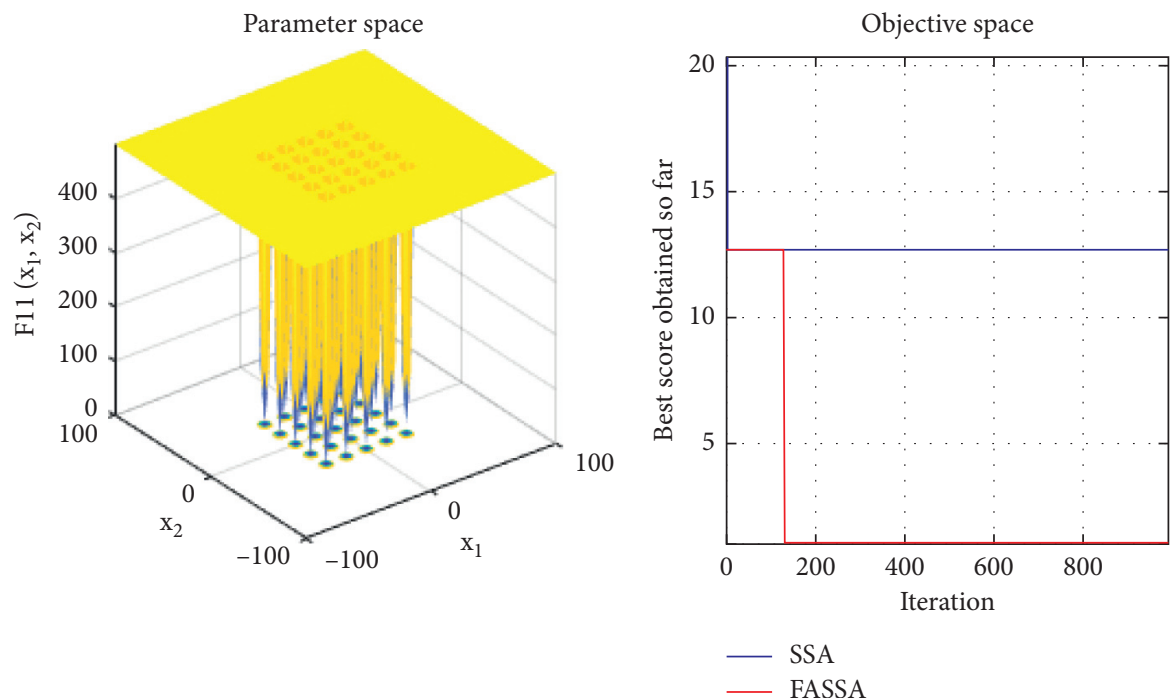

(a)

(b)

Figure 12: Experimental results of the test function $F_{11}$.

Step 7: run the network based on the weights and thresholds that have been allocated, and the gradient descent method is used to reversely propagate the error to continuously update and correct its value. Finally, the stop condition is reached, and the evaluation results are output.

The steps of FASSA optimization of BP neural networks can be summarized as the pseudo-code shown in Algorithm 1.

4.3. Constructing the Evaluation Model Based on FASSA-BP Algorithm. This section applies the improved FASSA-BP algorithm to construct the IMCM evaluation model of BP neural network based on FASSA.
4.3.1. Construction of the Experimental Environment. The experimental environment is as follows:

(1) Hardware aspects: Intel ${ }^{\circledR}$ Core $^{\mathrm{TM}}$ i5-4210U CPU, 4 GB memory capacity.

(2) Software aspects: Windows 10 64-bit version, Matlab 2017b software platform.

\subsubsection{Design of the Network Structure}

(1) Design of Input Layer Neurons. This paper takes the constructed secondary evaluation index as the input of the network model. In this paper, 20 secondary evaluation indexes 

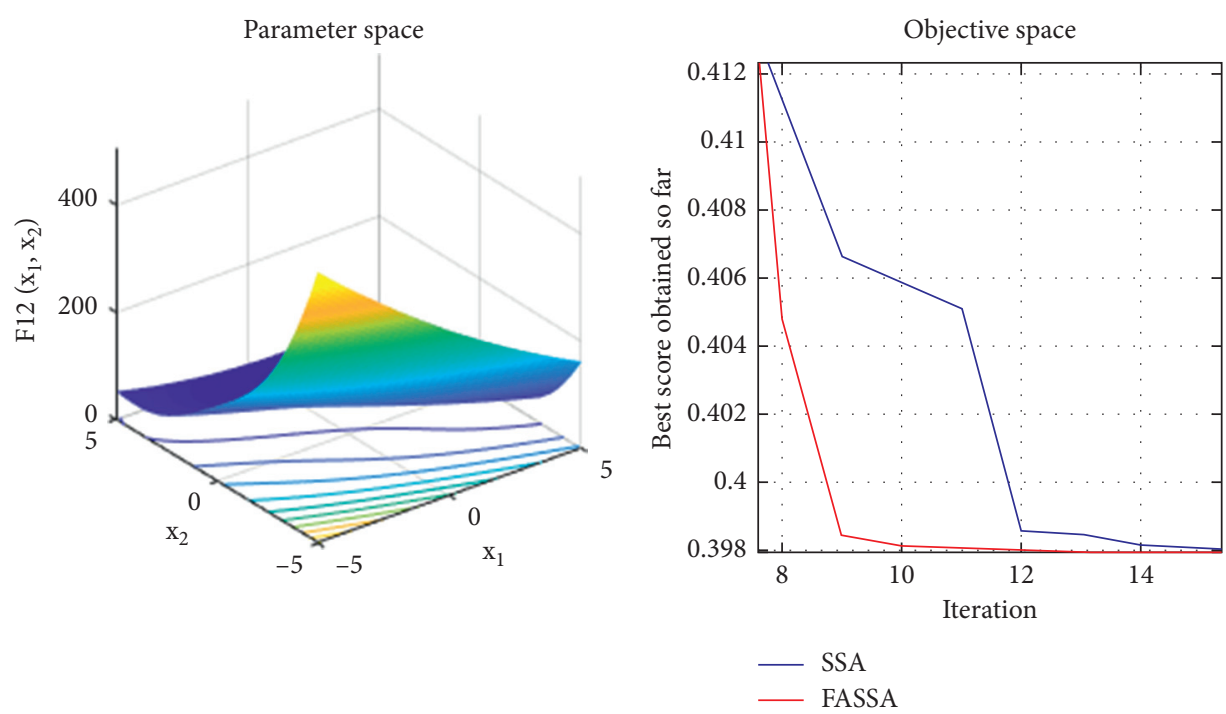

(a)

(b)

FIgURE 13: Experimental results of the test function $F_{12}$.

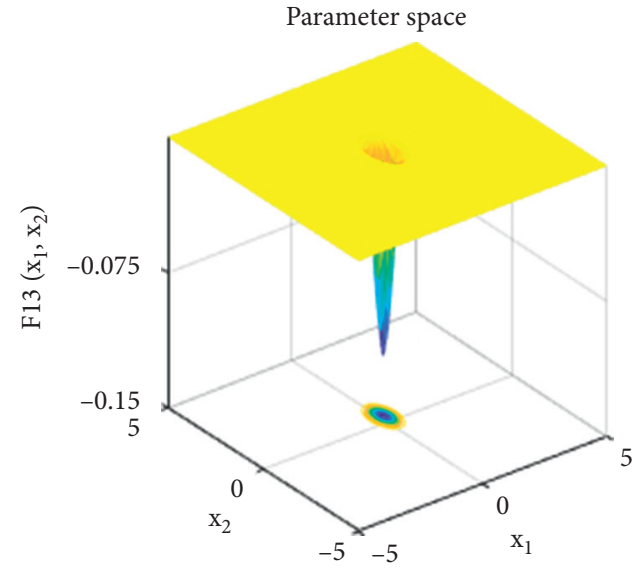

(a)

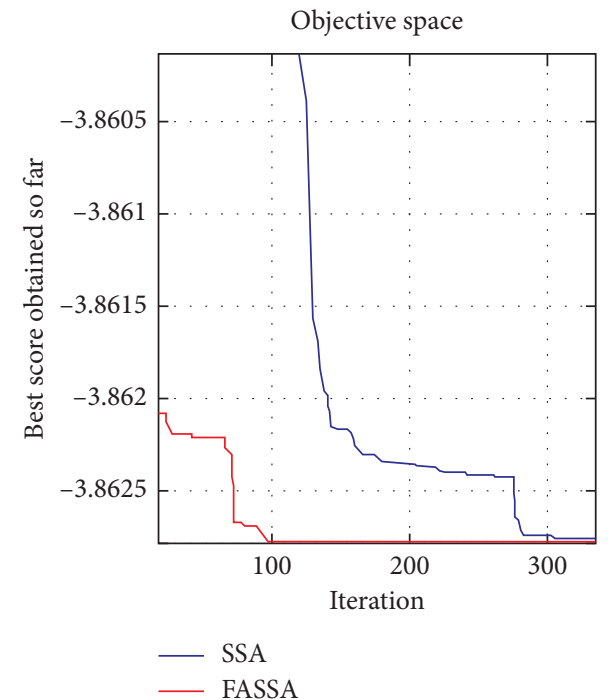

(b)

Figure 14: Experimental results of the test function $F_{13}$.

are used to measure the maturity of enterprise intelligent manufacturing capability. Therefore, the number of neurons in the input layer of the network is 20 , and the vector of the input layer is expressed as $X=\left(x_{1}, x_{2}, x_{3}, \ldots, x_{20}\right)$.

(2) Design of Hidden Layer Neurons. For the setting of hidden layer nodes, this paper adopts the classical method of computation, $S=\sqrt{(n+m)}+a$ (where $a$ is a constant within $[1,10]$ and $n$ and $m$ are the numbers of input layer and output layer) [33]. It is proved that when the number of hidden layer nodes is 5 , the model achieves the best state.

(3) Design of Output Layer Neurons. This paper takes the maturity score of intelligent manufacturing capability as the output layer of the network. Since there is only one output vector, the number of nodes in the output layer of the network structure in this experiment is 1 , and the output layer vector is expressed as $Y=\left(y_{1}\right)$.

Therefore, this paper builds a network with a structure of 20-5-1, as shown in Figure 17. Among them, $W_{1}=\left(\omega_{11}, \omega_{12}, \omega_{13}, \ldots, \omega_{120}\right)$ and $W_{2}=\left(\omega_{21}, \omega_{22}, \ldots, \omega_{25}\right)$ are, respectively, the weights between input layer and hidden layer, and between hidden layer and output layer; the hidden layer vector is set to $H_{1}=\left(h_{11}, h_{12}, \ldots, h_{15}\right)$.

(4) Determination and Selection of Parameters and Transfer Function. Considering that the evaluation objects in this paper have high accuracy requirements, the basic parameters of the network model are set as follows: learning rate is 0.01 , network training is 1000 times, and target error is $10^{-7}$. 


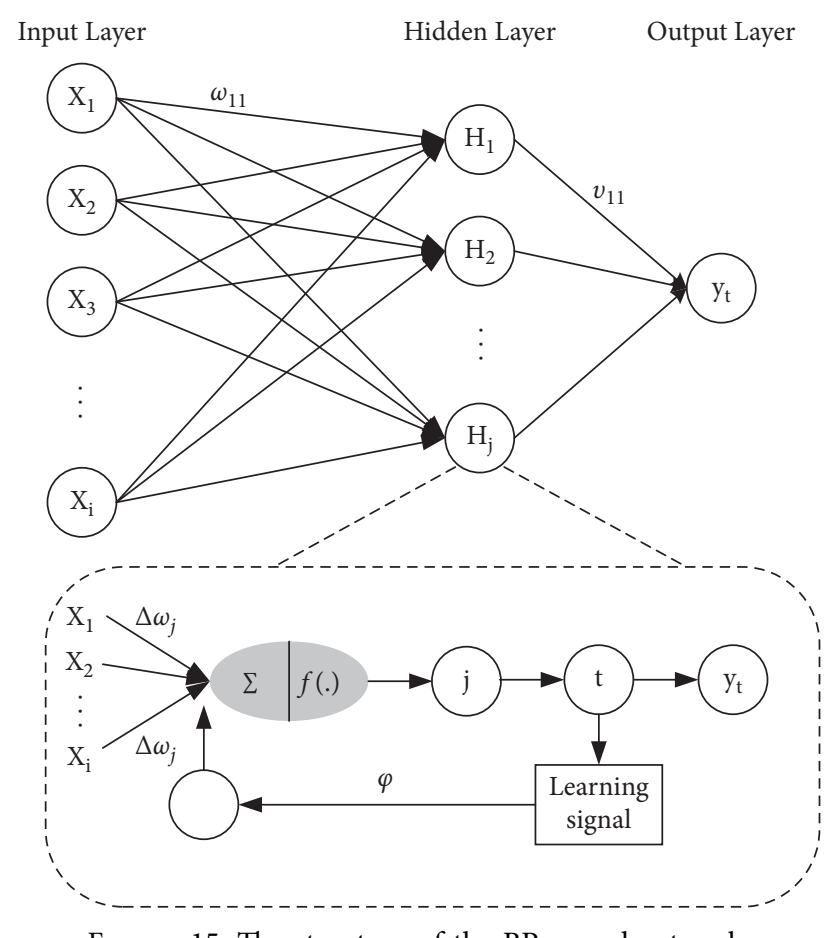

Figure 15: The structure of the BP neural network.

tansig and purelin act as hidden layer and output layer functions, respectively.

4.3.3. Selection of Network Performance Indicators. To test the performance of the evaluation model, this paper analyzes and compares the performance of FASSA-BP, SSA-BP, PSO$\mathrm{BP}$, and $\mathrm{BP}$ algorithms in terms of the accuracy and reliability of the model prediction results. In order to enhance the scientific nature of the evaluation of the forecast results, multiple performance metrics are used to evaluate the accuracy and stability of IMCM evaluation model results [34-38].

The performance analysis indexes used in this paper are as follows:

$$
\begin{aligned}
& \text { MRE }=\frac{1}{n} \sum_{i=1}^{n}\left|\frac{y_{i}-\hat{y}_{i}}{y_{i}}\right|, \\
& \mathrm{MSE}=\frac{1}{n} \sum_{i=1}^{n}\left(y_{i}-\hat{y}_{i}\right)^{2}, \\
& \mathrm{MAE}=\frac{1}{n} \sum_{i=1}^{n}\left|y_{i}-\hat{y}_{i}\right|,
\end{aligned}
$$

$$
\begin{aligned}
\mathrm{RMSE} & =\sqrt{\frac{1}{n} \sum_{i=1}^{n}\left(y_{i}-\hat{y}_{i}\right)^{2}}, \\
R^{2} & =\frac{\sum_{i=1}^{n}\left(y_{i}-\bar{y}\right)\left(\hat{y}_{i}-\tilde{y}\right)}{\sqrt{\sum_{i=1}^{n}\left(y_{i}-\bar{y}\right)^{2}} \sum_{i=1}^{n}\left(\hat{y}_{i}-\tilde{y}\right)^{2}}
\end{aligned} .
$$

In the above analysis index formulas, $n$ represents the number of samples, $y_{i}$ represents the true value of the sample, and $\hat{y}_{i}$ represents the model output value of the sample. The closer the evaluation indexes MRE, MSE, MAE, and RMSE are to 0 , the smaller the evaluation error is, and the stronger the evaluation ability of the model is. The value range of $R^{2}$ is $[0,1]$. The closer $R^{2}$ is to 1 , the better the evaluation effect is.

4.3.4. Expected Output Value of the Sample Data. The expected output value $E_{h}(h=1,2, \ldots, n)$ represents the true value of the enterprise intelligent manufacturing capability maturity score. The calculation of the expected output value of the sample needs to calculate the weight of the evaluation index and the score of the evaluation index. Because the topic of the questionnaire is designed according to the 20 secondary evaluation indexes above, the score of the secondary evaluation index is the sample data recovered. Arithmetic average method [39] was used to calculate the 


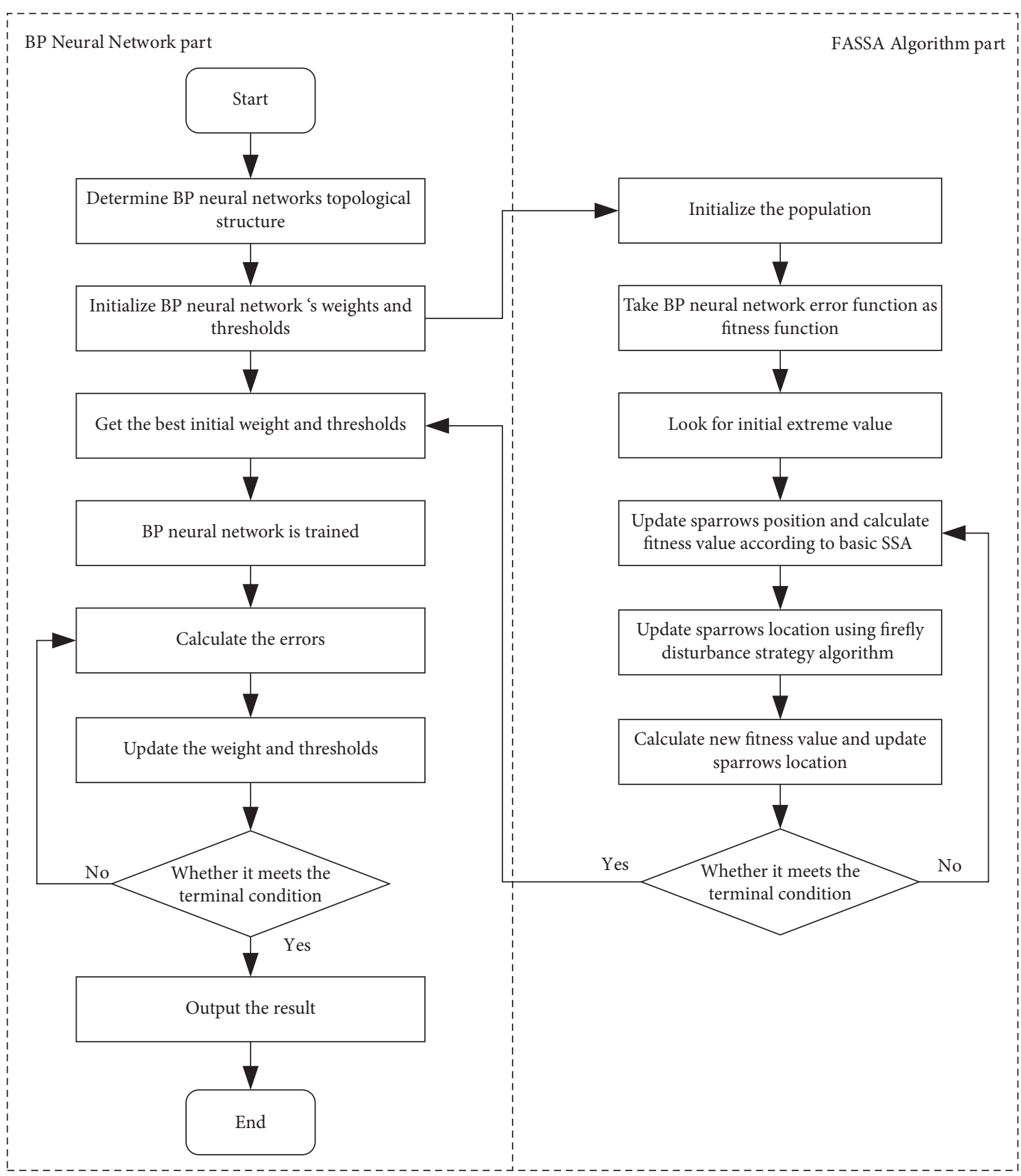

FIGURE 16: Flowchart of the BP neural network optimized by FASSA.

weight of primary index and the score of primary index, which were recorded as $H_{t}$ and $F_{t}$, respectively. The primary index was recorded as $B_{t}(t=1,2, \ldots, 10)$, and the secondary index was recorded as $C_{k}(k=1,2, \ldots, 20)$. The formula was as follows:

$$
\begin{aligned}
& H_{t}=\frac{\text { number of secondary indicators } C \text { corresponding to } B t}{20} \quad(t=1,2, \ldots, 10), \\
& F_{t}=\frac{\text { sum of scores of secondary indicators } C \text { corresponding to } B t}{\text { number of secondary indicators } C \text { corresponding to } \mathrm{Bt}} \quad(t=1,2, \ldots, 10) .
\end{aligned}
$$




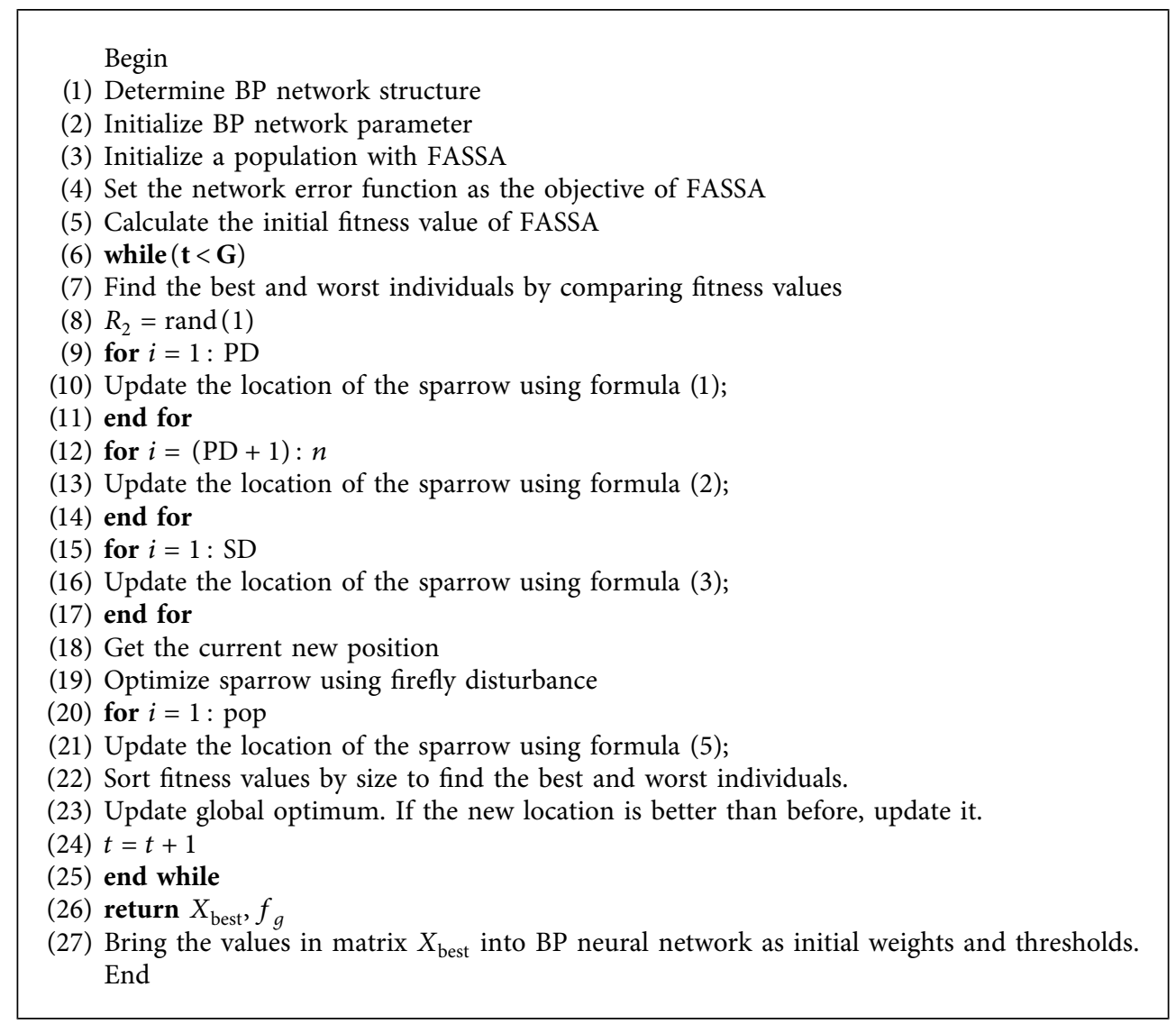

Algorithm 1: Pseudo-code of FASSA-BP algorithm.

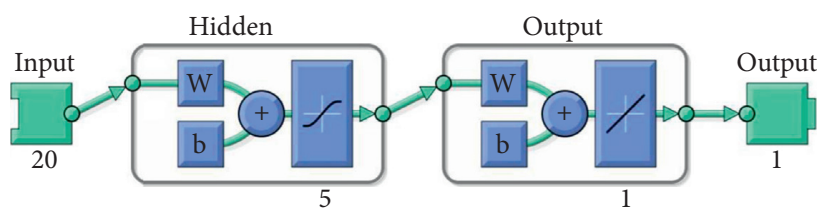

FIGURE 17: Network structure diagram of the model.

We use formula (11) to calculate the score of the firstlevel evaluation index and then multiply the score of each index in the sample data by the corresponding index weight with method of weighted mean. Finally, the expected output value $E_{h}$ of each sample is obtained by adding. The calculation formula is as follows:

$$
E_{h}=F_{1} * H_{1}+F_{2} * H_{2}+\cdots+F_{t} * H_{t}, \quad(t=1,2, \ldots, 10)
$$

The expected output value of the sample data is shown in Table 2.

4.3.5. IMCM Classification. Some intelligent manufacturing enterprises in China are selected for field visits to understand the actual situation of intelligent manufacturing enterprises in detail. At the same time, referring to the division of intelligent manufacturing capability maturity in the reports of White Paper on Intelligent
Manufacturing Capability Maturity Model and German Industry 4.0 Maturity Index, the intelligent manufacturing capability maturity of intelligent manufacturing enterprises is divided into five grades. The interval number of $(0,100)$ is used to divide the grades. The corresponding score intervals from low to high are $(0,30],(30,50],(50,70),(70,90),(90,100)$. The IMCM classification used in this paper is shown in Figure 18.

4.4. Evaluation Steps. The specific evaluation process of the IMCM evaluation model is shown in Figure 19.

Step 1: data acquisition and preprocessing: data are acquired and preprocessed according to the constructed evaluation index system to design the questionnaire, and then the questionnaire is distributed to the enterprise to collect data. Min-max method is used to normalize the recovered sample data. 


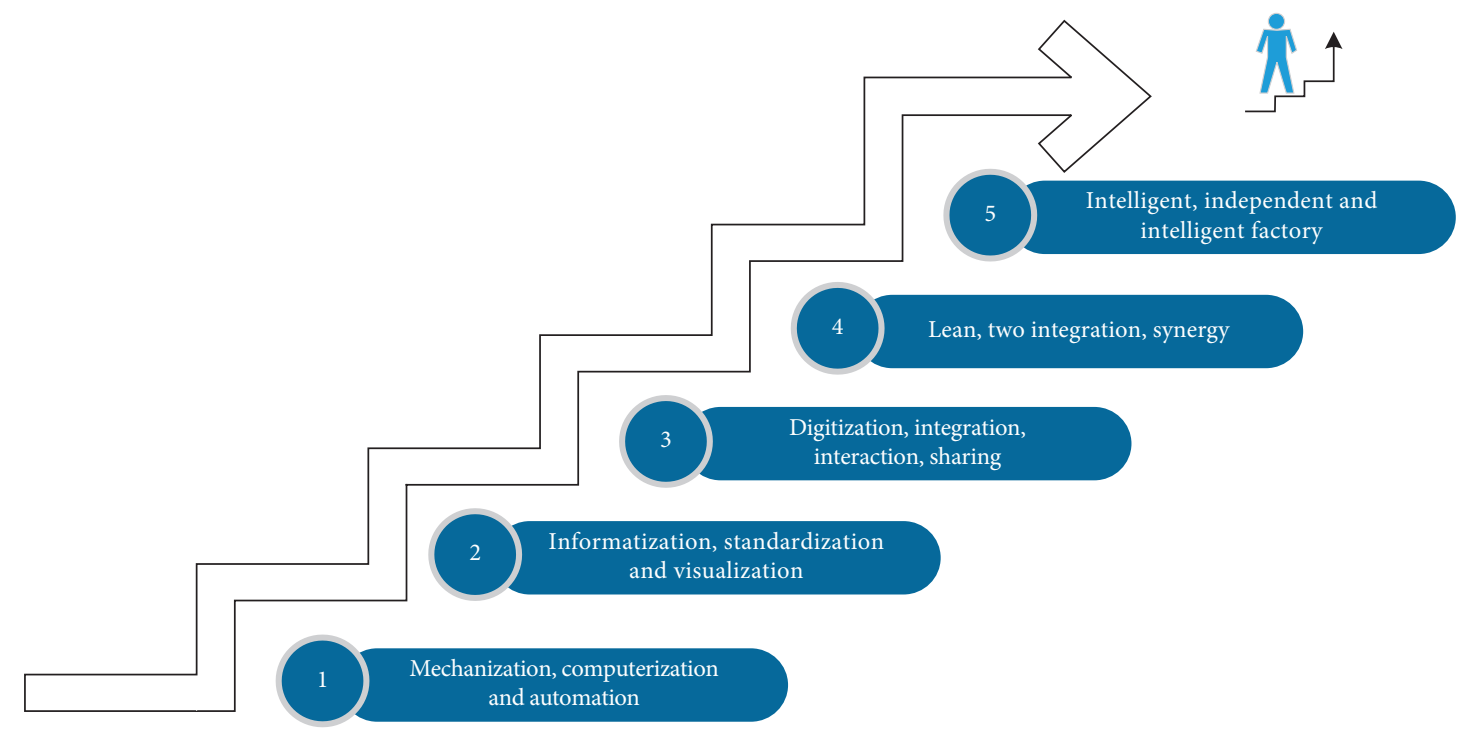

FIGURE 18: The level of enterprise intelligent manufacturing capability maturity.

Step 2: calculation of the sample expected output value: the hierarchical calculation method is adopted. First, the arithmetic average method [39] is used to calculate the first-level index weight and the first-level index score according to the recovered sample data, namely, the second level index score. Then, the weighted average method [40] is used to calculate the expected output value of the sample.

Step 3: determination of the experimental environment and model network structure: the experimental environment is introduced, and the numbers of input layer, output layer, and hidden layer of the model network structure are determined.

Step 4: comparison of neural network training and testing of different models: cross-validation experiments are conducted based on SSA-BP and PSO-BP models with the same population size and evolution times and the best population size and evolution times, respectively.

Step 5: analysis of results: the experimental results of the sample data of the test set are analyzed, and finally targeted suggestions for improvement are put forward for intelligent manufacturing enterprises.

\section{Empirical Analysis}

5.1. Application Object Profile. L International Technology Company Limited (hereinafter referred to as L Enterprise) is a well-known company in the manufacturing industry of China. Its main business field is battery manufacturing, and the products it produces and develops occupy an important position in the market of this field. The establishment of regional production bases at home and abroad constitutes a strong and advanced manufacturing and R\&D capability of the enterprise. However, as a large battery manufacturer, $\mathrm{L}$ Enterprise has many subsidiaries in different regions.
Although all the companies are developing intelligent manufacturing, due to the imbalance of economic development and scientific and technological level between regions, the development level of intelligent manufacturing differs between different subsidiaries. It is urgent to clarify the level of intelligent manufacturing of subsidiaries, which is consistent with the research background selected in this paper and can accurately obtain the evaluation index data needed in this study. Therefore, the enterprise is selected as the actual case of the study.

\subsection{Data Acquisition and Data Preprocessing}

5.2.1. Data Acquisition. The data of this paper is collected in the form of questionnaire survey and expert interview, and the sample data is obtained according to the following four steps:

(1) According to the quantitative method of evaluation index introduced above, a preliminary questionnaire survey is designed by using the constructed evaluation index to recover the quantitative index data. Through the questionnaire survey, these $20 \mathrm{sec}-$ ondary evaluation indexes are quantified into the determined sample data as the input layer data of the network.

(2) The advanced manufacturing enterprises are selected, through research and interviews with the management personnel of the enterprise and the staff of the main business department; the collected opinions are summarized; the questionnaire is modified according to the refined suggestions, in order to set the appropriate questionnaire questions, options etc.; and finally the questionnaire is determined.

(3) In order to improve the quality of the questionnaire, some questionnaires were distributed before the 


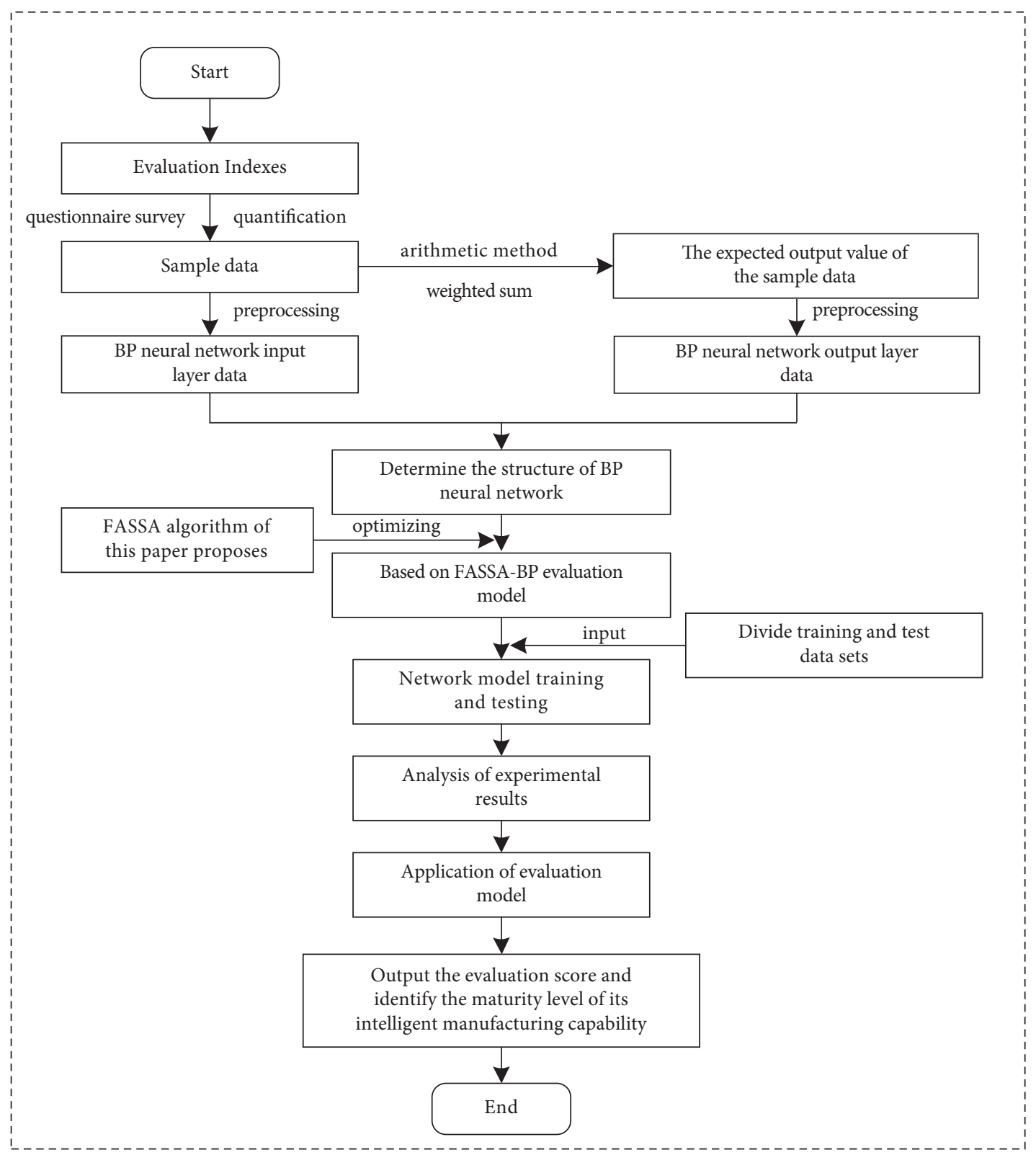

FIGURE 19: FASSA-BP model evaluation flowchart.

formal issuance of the questionnaire and according to the feedback in advance to improve the questionnaire.

(4) Select the subsidiary of L Enterprise as the research object, and obtain data through enterprise research and questionnaire. The collected data help to support case studies in detail by understanding the development level of intelligent manufacturing enterprises in each evaluation index. A total of 536 questionnaires were returned, including 40 invalid questionnaires.

5.2.2. Division of Datasets. This paper collects 496 valid questionnaires and divides them into 441 data for network model training, which is the training set data. Then, samples are selected from the remaining 55 sample data as the test set. Finally, 5 subcompany data are left, and the trained network model is used to evaluate the application.

5.2.3. Preprocessing of the Data. Considering that the magnitude of the original data is quite different, which has a great impact on the network training process and increases the difficulty of training, in order to better analyze the data and improve the training efficiency of the model, before the neural network training, Min - max method that belongs to the normalization method is used to sort out the sample data and its expected output value [41]. Because the evaluation indexes in this paper are positive, the normalization only considers the positive situation, and the calculation formula is shown in 


$$
X^{*}=\frac{X-X_{\min }}{X \min _{\max }} .
$$

The original data obtained by sorting out the collected questionnaire are shown in Table 3 . The original data are processed in advance by (16) to obtain the input sample data as shown in Table 4. Finally, the data processed in advance is input into the constructed evaluation model to ensure that the trained model can accurately evaluate the maturity of enterprise intelligent manufacturing capability.

\subsubsection{Calculation of the Expected Output Value of the Sample} Data. The data obtained in Section 5.2.1 is the sample data quantified by the evaluation index, that is, the input layer data of the network model. However, in order to use the network model for evaluation research, the expected output value of the sample data is also needed, that is, the ability maturity evaluation score, as the output layer data of the network model.

From Section 4.3.4, we can get the weight of the evaluation index and its score, and then the weighted sum of the two can get the expected output value of the sample data.

(1) Calculating the Weight of Evaluation Index. According to Section 4.3.4, the weight of evaluation index is calculated by arithmetic average method and weighted average method. According to (13), the weight of first-level evaluation index can be obtained:

$$
\begin{aligned}
& H_{1}=H_{2}=H_{4}=H_{5}=\frac{1}{10}, \\
& H_{3}=\frac{7}{20}, \\
& H_{6}=H_{7}=H_{8}=H_{9}=H_{10}=\frac{1}{20} .
\end{aligned}
$$

(2) Calculating Scores of Evaluation Indicators. Through the quantification of evaluation indexes, it can be seen that, in the questionnaire survey table, each question reflects the situation of each secondary evaluation index, so the sample data collected by the questionnaire correspond to the score of the secondary evaluation index. Then, according to (14), the first-level evaluation index score is calculated.

(3) Calculating the Expected Output of the Sample Data. Finally, the expected output value of each sample data can be obtained by weighted summation of the first-level evaluation index score and its corresponding index weight. The expected output value of the sample data is shown in Table 5.

\subsection{Enterprise Intelligent Manufacturing Capability Maturity Evaluation}

5.3.1. Comparison of Evaluation Model Training and Testing. The population size and the number of iterations have a great influence on the optimization algorithm to find the best solution. Therefore, this paper designs comparison experiments from the two perspectives of the same population size and number of iterations, and the optimal population size and number of iterations, respectively, and selects the BP model, PSO-BP model, SSA-BP model, and FASSA-BP model proposed in this paper for performance comparison.

Experiment 1. Performance comparison based on the same population size and iterations.

In Experiment 1, in order to reduce the influence of initial parameters on the performance of the algorithm, the settings of the same parameters are guaranteed to be the same. Because the comparison of several algorithm models is based on BP neural network, the BP neural network parameters are set according to Section 4.3.2. The key parameters in PSO-BP model, SSA-BP model, and FASSA-BP model are set as shown in Table 6 .

After setting the parameters of each model, the preprocessed sample data are input into FASSA-BP model, SSA-BP model, PSO-BP model, and BP model for training and testing. Firstly, the training samples are input into the four models to train. After the training is completed, it enters the test stage. After several iterations, the comparison results of the evaluation value and the real value of each model test set sample are obtained as shown in Figure 20, and the relative error results of the expected output value of each model test set sample data and the actual output value of the model are obtained as shown in Figure 21.

Considering the different running results of neural network algorithm and intelligent optimization algorithm, the four models are run 30 times, respectively, and the average values of the experimental results of performance evaluation indexes of different models are compared, as shown in Table 7.

Combining Figures 20 and 21 and Table 7, we can see that the evaluation accuracy of the FASSA-BP model proposed in this paper is significantly higher than that of the basic SSA-BP model, PSO-BP model, and traditional BP model, and the value of the correlation coefficient $R 2$ is closer to 1 than that of the other three models, which verifies that the FASSA-BP model can be better applied to solve the maturity evaluation problem of intelligent manufacturing capability.

Experiment 2. Performance comparison based on optimal population size and iterations.

In this paper, by selecting the test function to set different population size and iteration number, the optimal population size and iteration number of PSO algorithm, SSA, and FASSA are obtained to achieve the optimal fitness value, respectively, as shown in Table 8 .

In order to further test the superiority of FASSA-BP model, the parameters of FASSA-BP model, SSA-BP model, and PSO-BP model under the optimal fitness are selected as the best parameter settings, and their model performance is analyzed by experiments. In the same experiment, after the parameter setting is completed, the preprocessed sample data are input to FASSA-BP model, 
TABle 3: Original data from questionnaires.

\begin{tabular}{lcccccccccccccccccccc}
\hline Samples & C1 & C2 & C3 & C4 & C5 & C6 & C7 & C8 & C9 & C10 & C11 & C12 & C13 & C14 & C15 & C16 & C17 & C18 & C19 & C20 \\
\hline S1 & 60 & 60 & 80 & 60 & 80 & 40 & 60 & 80 & 60 & 60 & 80 & 80 & 80 & 80 & 80 & 40 & 60 & 80 & 100 & 40 \\
S2 & 80 & 60 & 60 & 60 & 60 & 40 & 40 & 40 & 40 & 40 & 20 & 60 & 60 & 40 & 40 & 60 & 60 & 60 & 40 & 20 \\
S3 & 60 & 20 & 20 & 20 & 40 & 60 & 40 & 40 & 0 & 80 & 0 & 80 & 60 & 40 & 100 & 40 & 60 & 60 & 0 & 0 \\
S4 & 0 & 20 & 60 & 60 & 60 & 40 & 40 & 40 & 80 & 80 & 80 & 60 & 60 & 0 & 60 & 60 & 60 & 40 & 40 & 40 \\
S5 & 40 & 40 & 60 & 20 & 80 & 80 & 100 & 100 & 100 & 100 & 20 & 20 & 40 & 40 & 40 & 80 & 20 & 20 & 80 & 60 \\
$\ldots$ & $\ldots$ & $\ldots$ & $\ldots$ & $\ldots$ & $\ldots$ & $\ldots$ & $\ldots$ & $\ldots$ & $\ldots$ & $\ldots$ & $\ldots$ & $\ldots$ & $\ldots$ & $\ldots$ & $\ldots$ & $\ldots$ & $\ldots$ & $\ldots$ & $\ldots$ & $\ldots$ \\
S487 & 40 & 100 & 0 & 40 & 60 & 20 & 0 & 20 & 80 & 20 & 60 & 40 & 40 & 20 & 20 & 60 & 40 & 80 & 80 & 60 \\
S488 & 20 & 80 & 20 & 60 & 40 & 40 & 60 & 40 & 80 & 20 & 20 & 40 & 60 & 80 & 0 & 60 & 100 & 40 & 0 & 0 \\
S489 & 0 & 20 & 40 & 60 & 20 & 40 & 40 & 40 & 40 & 0 & 20 & 20 & 40 & 20 & 20 & 20 & 40 & 40 & 60 & 0 \\
S490 & 100 & 60 & 40 & 80 & 80 & 60 & 60 & 60 & 40 & 60 & 40 & 60 & 0 & 40 & 20 & 20 & 80 & 20 & 100 & 60 \\
S491 & 40 & 100 & 60 & 80 & 0 & 40 & 80 & 100 & 20 & 40 & 20 & 80 & 40 & 40 & 0 & 20 & 60 & 100 & 80 & 100 \\
S492 & 100 & 80 & 100 & 60 & 0 & 100 & 100 & 100 & 40 & 100 & 60 & 100 & 100 & 60 & 40 & 60 & 60 & 20 & 20 & 80 \\
S493 & 40 & 0 & 80 & 20 & 80 & 20 & 40 & 60 & 40 & 20 & 60 & 100 & 80 & 60 & 80 & 80 & 0 & 60 & 60 & 80 \\
S494 & 80 & 60 & 40 & 80 & 60 & 60 & 20 & 100 & 80 & 60 & 80 & 80 & 80 & 60 & 60 & 80 & 60 & 40 & 60 & 40 \\
S495 & 100 & 100 & 40 & 80 & 40 & 60 & 80 & 20 & 100 & 0 & 20 & 40 & 0 & 100 & 80 & 80 & 40 & 80 & 80 & 100 \\
S496 & 80 & 100 & 40 & 60 & 100 & 100 & 60 & 80 & 60 & 80 & 80 & 80 & 80 & 80 & 60 & 80 & 80 & 60 & 100 & 0 \\
\hline
\end{tabular}

TABLE 4: Input sample data after preprocessing.

\begin{tabular}{lcccccccccccccccccccc}
\hline Samples & C1 & C2 & C3 & C4 & C5 & C6 & C7 & C8 & C9 & C10 & C11 & C12 & C13 & C14 & C15 & C16 & C17 & C18 & C19 & C20 \\
\hline S1 & 0.6 & 0.6 & 0.8 & 0.6 & 0.8 & 0.4 & 0.6 & 0.8 & 0.6 & 0.6 & 0.8 & 0.8 & 0.8 & 0.8 & 0.8 & 0.4 & 0.6 & 0.8 & 1.0 & 0.4 \\
S2 & 0.8 & 0.6 & 0.6 & 0.6 & 0.6 & 0.4 & 0.4 & 0.4 & 0.4 & 0.4 & 0.2 & 0.6 & 0.6 & 0.4 & 0.4 & 0.6 & 0.6 & 0.6 & 0.4 & 0.2 \\
S3 & 0.6 & 0.2 & 0.2 & 0.2 & 0.4 & 0.6 & 0.4 & 0.4 & 0.0 & 0.8 & 0.0 & 0.8 & 0.6 & 0.4 & 1.0 & 0.4 & 0.6 & 0.6 & 0.0 & 0.0 \\
S4 & 0.0 & 0.2 & 0.6 & 0.6 & 0.6 & 0.4 & 0.4 & 0.4 & 0.8 & 0.8 & 0.8 & 0.6 & 0.6 & 0.0 & 0.6 & 0.6 & 0.6 & 0.4 & 0.4 & 0.4 \\
S5 & 0.4 & 0.4 & 0.6 & 0.2 & 0.8 & 0.8 & 1.0 & 1.0 & 1.0 & 1.0 & 0.2 & 0.2 & 0.4 & 0.4 & 0.4 & 0.8 & 0.2 & 0.2 & 0.8 & 0.6 \\
$\ldots$ & $\ldots$ & $\ldots$ & $\ldots$ & $\ldots$ & $\ldots$ & $\ldots$ & $\ldots$ & $\ldots$ & $\ldots$ & $\ldots$ & $\ldots$ & $\ldots$ & $\ldots$ & $\ldots$ & $\ldots$ & $\ldots$ & $\ldots$ & $\ldots$ & $\ldots$ & $\ldots$ \\
S487 & 0.4 & 1.0 & 0.0 & 0.4 & 0.6 & 0.2 & 0.0 & 0.2 & 0.8 & 0.2 & 0.6 & 0.4 & 0.4 & 0.2 & 0.2 & 0.6 & 0.4 & 0.8 & 0.8 & 0.6 \\
S488 & 0.2 & 0.8 & 0.2 & 0.6 & 0.4 & 0.4 & 0.6 & 0.4 & 0.8 & 0.2 & 0.2 & 0.4 & 0.6 & 0.8 & 0.6 & 0.0 & 1.0 & 0.4 & 0.0 & 0.0 \\
S489 & 0.0 & 0.2 & 0.4 & 0.6 & 0.2 & 0.4 & 0.4 & 0.4 & 0.4 & 0.0 & 0.2 & 0.2 & 0.4 & 0.2 & 0.2 & 0.2 & 0.4 & 0.4 & 0.6 & 0.0 \\
S490 & 1.0 & 0.6 & 0.4 & 0.8 & 0.8 & 0.6 & 0.6 & 0.6 & 0.4 & 0.6 & 0.4 & 0.6 & 0.0 & 0.4 & 0.2 & 0.2 & 0.8 & 0.2 & 1.0 & 0.6 \\
S491 & 0.4 & 1.0 & 0.6 & 0.8 & 0.0 & 0.4 & 0.8 & 1.0 & 0.2 & 0.4 & 0.2 & 0.6 & 0.4 & 0.4 & 0.0 & 0.2 & 0.6 & 1.0 & 0.8 & 1.0 \\
S492 & 1.0 & 0.8 & 1.0 & 0.6 & 0.0 & 1.0 & 1.0 & 1.0 & 0.2 & 1.0 & 0.6 & 1.0 & 1.0 & 0.6 & 0.4 & 0.6 & 06 & 0.2 & 0.2 & 0.8 \\
S493 & 0.4 & 0.0 & 0.8 & 0.2 & 0.8 & 0.2 & 0.4 & 0.6 & 0.4 & 0.2 & 0.6 & 1.0 & 0.8 & 0.6 & 0.8 & 0.8 & 0.0 & 0.6 & 0.6 & 0.8 \\
S494 & 0.8 & 0.6 & 0.4 & 0.8 & 0.6 & 0.6 & 0.2 & 1.0 & 0.8 & 0.6 & 0.8 & 0.8 & 0.8 & 0.6 & 0.6 & 0.8 & 0.6 & 0.4 & 0.6 & 0.4 \\
S495 & 1.0 & 1.0 & 0.4 & 0.8 & 0.4 & 0.6 & 0.8 & 0.2 & 1.0 & 0.0 & 0.2 & 0.4 & 0.0 & 1.0 & 0.8 & 0.8 & 0.4 & 0.8 & 0.8 & 1.0 \\
S496 & 0.8 & 0.8 & 0.4 & 0.6 & 1.0 & 1.0 & 0.6 & 0.8 & 0.6 & 0.8 & 0.8 & 0.8 & 0.8 & 0.8 & 0.6 & 0.8 & 0.8 & 0.6 & 1.0 & 0.0 \\
\hline
\end{tabular}

TABLE 5: Expected output value of the sample data.

\begin{tabular}{lccccc}
\hline Sample & $E$ & Sample & $E$ & Sample & $E$ \\
\hline$S_{1}$ & 68 & $\ldots$ & $\ldots$ & $S_{486}$ & 57 \\
$S_{2}$ & 49 & $\ldots$ & $\ldots$ & $S_{487}$ & 44 \\
$S_{3}$ & 41 & $S_{482}$ & 41 & $S_{488}$ & 43 \\
$S_{4}$ & 49 & $S_{483}$ & 62 & $S_{489}$ & 29 \\
$S_{5}$ & 57 & $S_{484}$ & 71 & $S_{490}$ & 54 \\
$\ldots$ & $\ldots$ & $S_{485}$ & 65 & $S_{491}$ & 55 \\
\hline
\end{tabular}

TABle 6: Parameter settings of model algorithms in Experiment 1.

\begin{tabular}{lccc}
\hline Arithmetic & $\begin{array}{c}\text { Population } \\
\text { size }\end{array}$ & $\begin{array}{c}\text { Iteration } \\
\text { times }\end{array}$ & $\begin{array}{c}\text { Individual } \\
\text { parameter }\end{array}$ \\
\hline PSO & 100 & 100 & $C 1=C 2=1.49445$ \\
SSA & 100 & 100 & P_percent $=0.2$ \\
FASSA & 100 & 100 & P_percent $=0.2$ \\
\hline
\end{tabular}

SSA-BP model, PSO-BP model, and BP model, respectively. After several iterations, the evaluation value of each model is obtained and compared with the real value, as shown in Figure 22. In addition, there is a relative error, as shown in Figure 23.

The FASSA-BP model, SSA-BP model, and PSO-BP model are set to the best population size and number of iterations, respectively. Because the running time of the three models is too long in the case of the best population size and number of iterations, the FASSA-BP model, SSA-BP model, and PSO-BP model are run 20 times, respectively, and the average value of the experimental data of each model in the performance evaluation index is calculated to analyze and compare the performance of these models, as shown in Table 9.

From the comparison between the evaluation value and the real value of the sample in Figure 22 test set, FASSA-BP evaluation model has better evaluation effect. It can be seen from Figure 23 that the relative error between the true value of FASSA-BP model and the actual network output value is the smallest. According to Table 10, although the SSA-BP model and the PSO-BP algorithm model are under the optimal population size and iteration number, from the perspective of 


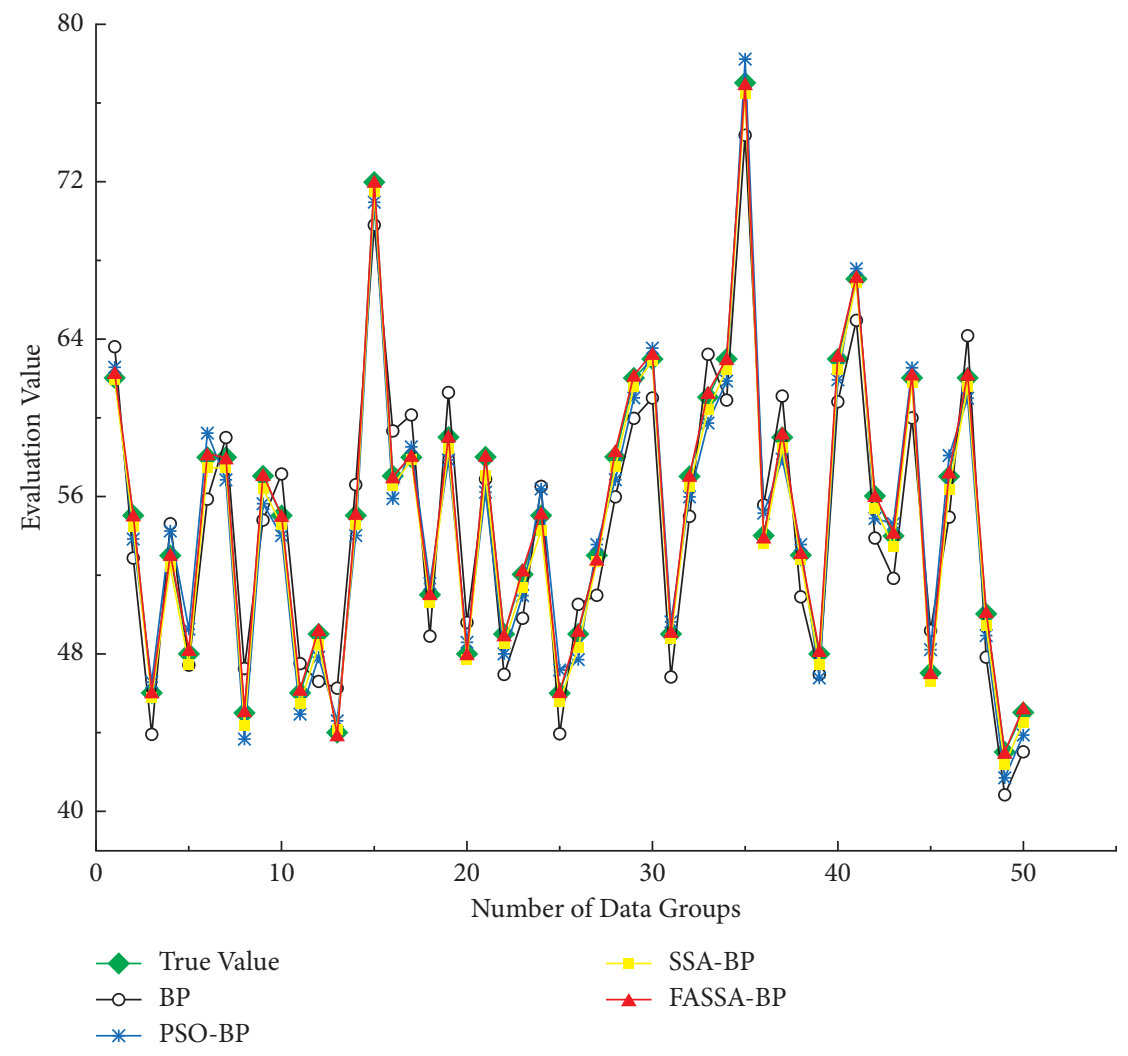

Figure 20: Comparison of test results of each model in Experiment 1.

evaluation accuracy, the FASSA-BP model is significantly better than the SSA-BP model, the PSO-BP model, and the traditional BP model, which further proves the superiority of the FASSA-BP model proposed in this paper.

5.3.2. Performance Analysis of the FASSA-BP Model. In order to analyze FASSA-BP model more accurately, regression analysis and relative error analysis of model evaluation value and expected output value of FASSA-BP model test set sample data are selected, as shown in Figure 24 and Table 10.

The regression analysis of the actual output value and the expected output value of the network (Figure 24) shows that regression coefficient R2 of FASSA-BP model is very close to 1 , which indicates that the evaluation effect of FASSA-BP model is very good.

From the comparison results of the relative error between the output value of the model and the real value of the sample in Table 10 test set, it can be concluded that the error of FASSA-BP evaluation model is small, and the classification results are completely consistent, which also shows that the comprehensive evaluation score of the model for the maturity of intelligent manufacturing capability is almost the same as the actual intelligent manufacturing capability of the enterprise, and it has certain accuracy and feasibility. It is further proved that the model can be used in the evaluation of the maturity of intelligent manufacturing capability.
5.3.3. Maturity Evaluation of Subsidiary Intelligent Manufacturing Capability. According to the different geographical distribution, the attribute values of the secondary evaluation indexes of five subsidiaries (the numbers of subsidiaries are $C 1, C 2, C 3, C 4, C 5)$ of the enterprise are selected as the sample data. The trained FASSA-BP evaluation model is used to calculate the evaluation value of the intelligent manufacturing capability maturity of each subsidiary. According to the classification of evaluation grades, the corresponding intelligent manufacturing capability maturity levels of the five subsidiaries are obtained, as shown in Table 11.

5.4. Empirical Result Analysis and Improvement Suggestions. By applying the evaluation model based on FASSA-BP algorithm proposed in this paper to practical cases and comparing it with other evaluation models, it can be seen that FASSA-BP model is more accurate and reliable than other models and can better deal with the maturity evaluation of enterprise intelligent manufacturing capability.

It can be seen from Table 11 that there is a certain gap in the maturity level of intelligent manufacturing capability of these five subsidiaries. Affected by their geographical location and the level of urban economic development, these subsidiaries have different performance in different evaluation indicators. Among them, $\mathrm{C} 4$ is the highest rating; the ability maturity score is 73 , which shows that the company's intelligent manufacturing capability is relatively mature. However, although the company's overall performance in the 


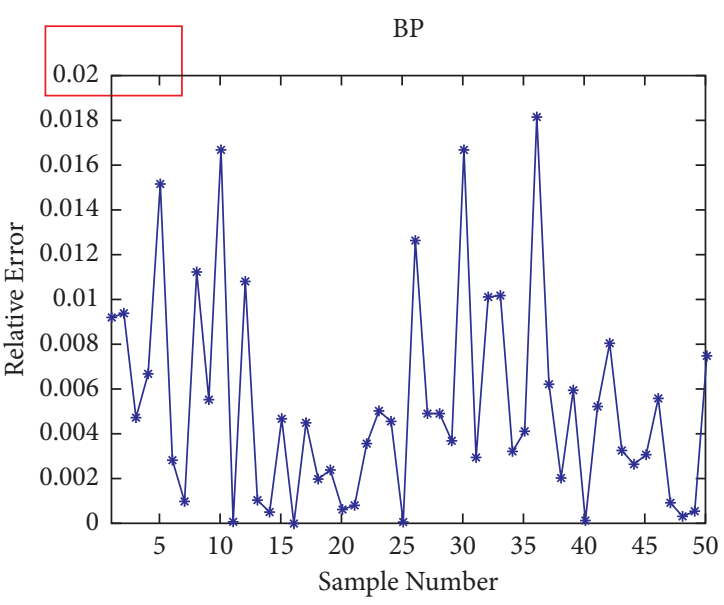

(a)

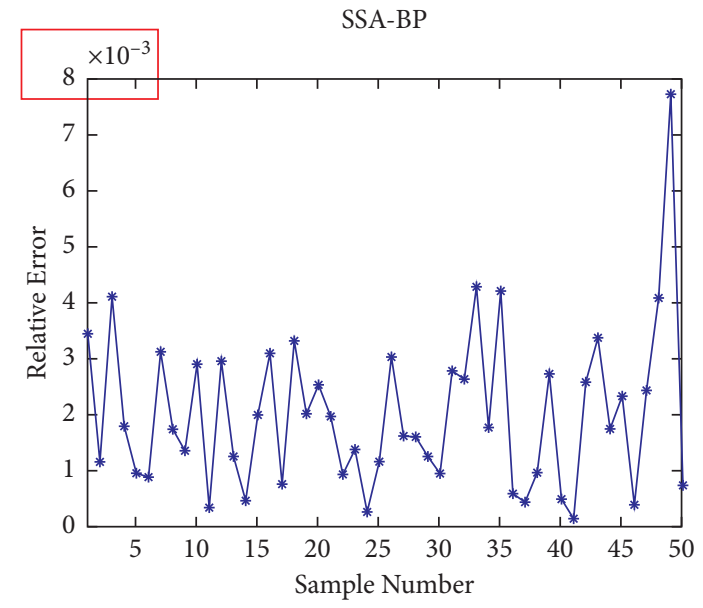

(c)

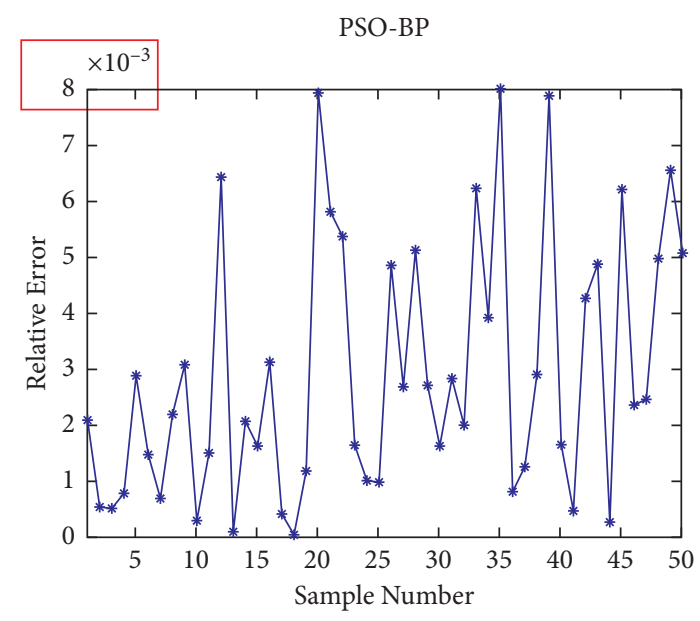

(b)

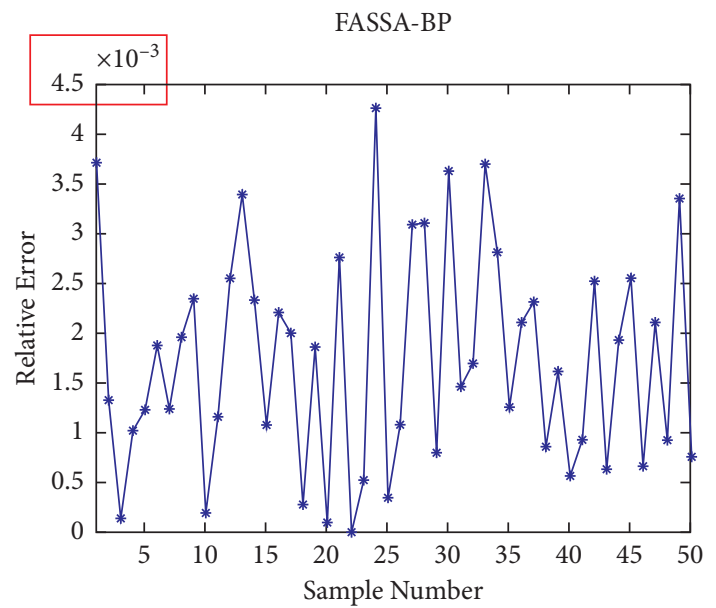

(d)

FIGURE 21: Relative error of each model test set sample in Experiment 1: (a) BP; (b) PSO-BP; (c) SSA-BP; (d) FASSA-BP.

TABLE 7: Average data of four model performance evaluation indexes in Experiment 1.

\begin{tabular}{lcccc}
\hline $\begin{array}{l}\text { Performance evaluation } \\
\text { index }\end{array}$ & BP & PSO-BP & SSA-BP & FASSA-BP \\
\hline MRE (\%) & 0.4845 & 0.3523 & 0.1781 & 0.1483 \\
MSE (\%) & 8.4504 & 5.8176 & 2.3848 & 1.7848 \\
MAE (\%) & 25.39 & 18.97 & 11.61 & 9.866 \\
RMSE & 0.2957 & 0.2459 & 0.1646 & 0.1294 \\
$R^{2}$ & 0.9824 & 0.9861 & 0.9995 & 0.9997 \\
\hline
\end{tabular}

TABLE 8: Optimal parameter settings for model algorithms in Experiment 2.

\begin{tabular}{lccc}
\hline Arithmetic & Population size & Iteration times & $\begin{array}{c}\text { Individual } \\
\text { parameter }\end{array}$ \\
\hline PSO & 200 & 1000 & $C 1=C 2=1.49445$ \\
SSA & 100 & 1000 & P_percent $=0.2$ \\
FASSA & 100 & 1000 & P_percent $=0.2$ \\
\hline
\end{tabular}

production process is good, the process management is still insufficient; the company needs to focus on the next improvement. Although $C 1, C 2$, and $C 3$ have the same rating, they have different performance in evaluation indicators. For example, $C 1$ 's advantage lies in network management, but the index to be improved is $5 \mathrm{~S}$ management, so the company should focus on its low maturity $5 \mathrm{~S}$ management in the future. One of the main reasons for the low level of $C 2$ capability is quality control. Therefore, the company should establish a quality management system to help enterprises control product quality by automatically collecting quality information in the production process. C3 scored relatively low in maturity of strategic management indicators, so the company should adjust its strategy to keep the development of intelligent manufacturing consistent with the company's development strategy. $C 5$, as the second level of evaluation, belongs to "intelligent manufacturing novice" and has a greater opportunity to enter the next maturity level. Its maturity evaluation is significant. Therefore, in the next step, the company should strengthen its organizational structure and mechanism construction. It can be seen from this that, for the maturity of intelligent manufacturing capability, different subsidiaries have differences in the evaluation index. Therefore, each company should pay more attention to the 


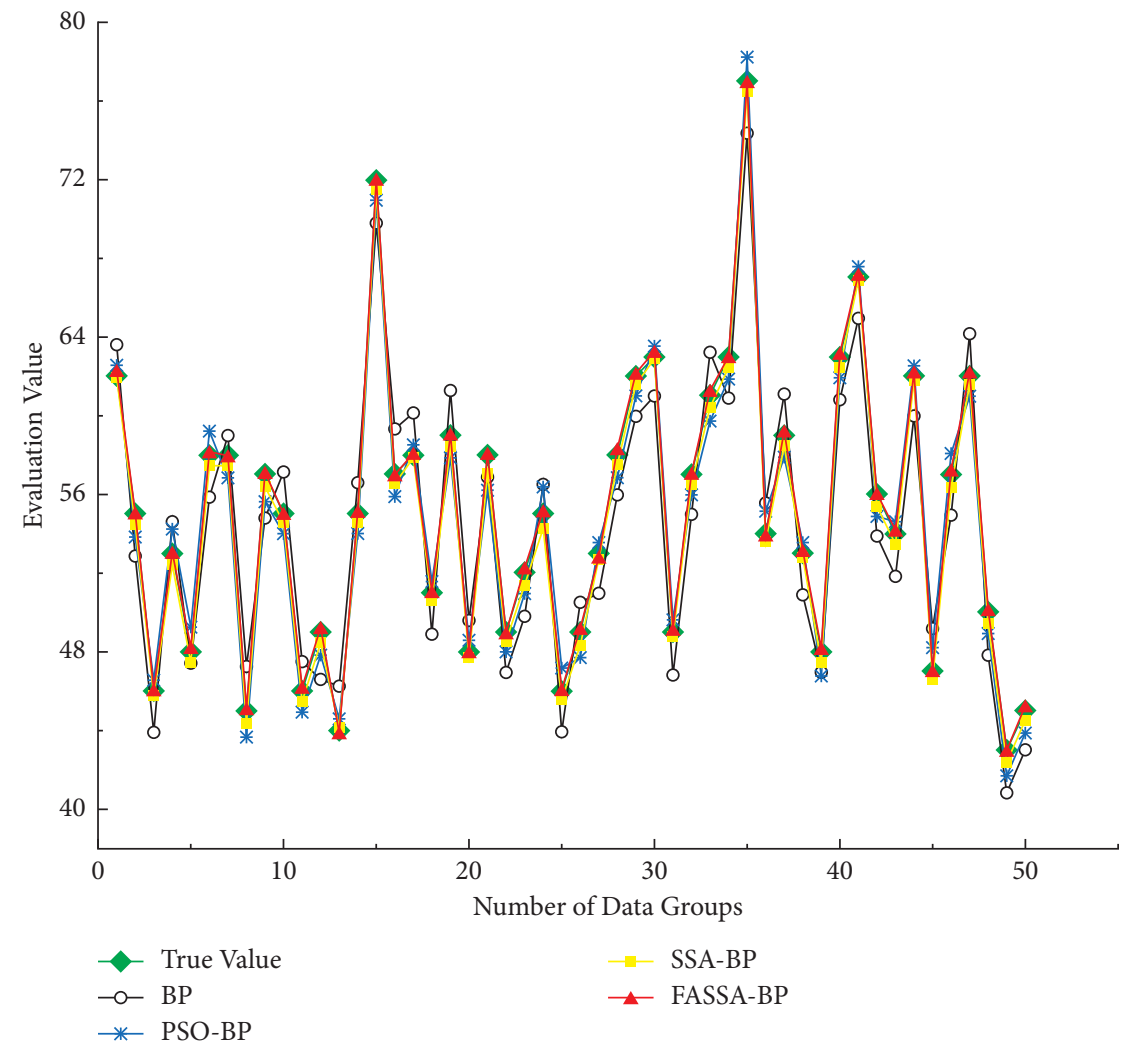

Figure 22: Comparison of test results of each model in Experiment 2.

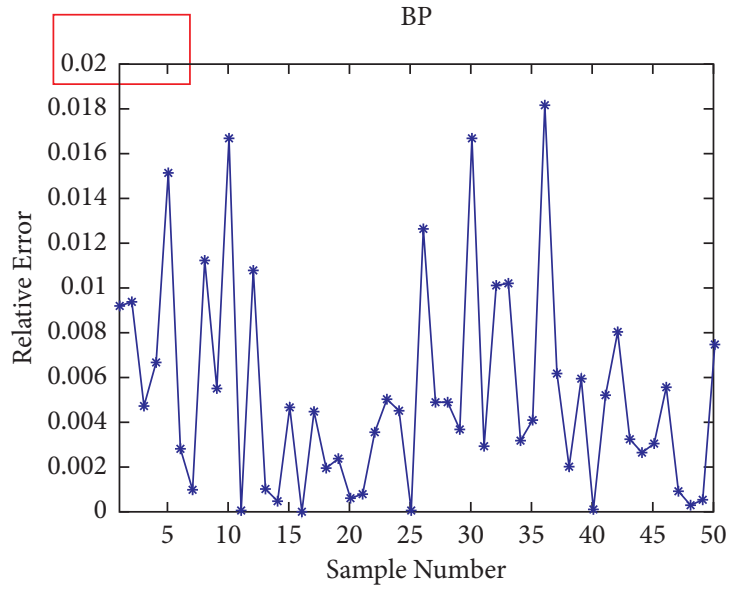

(a)

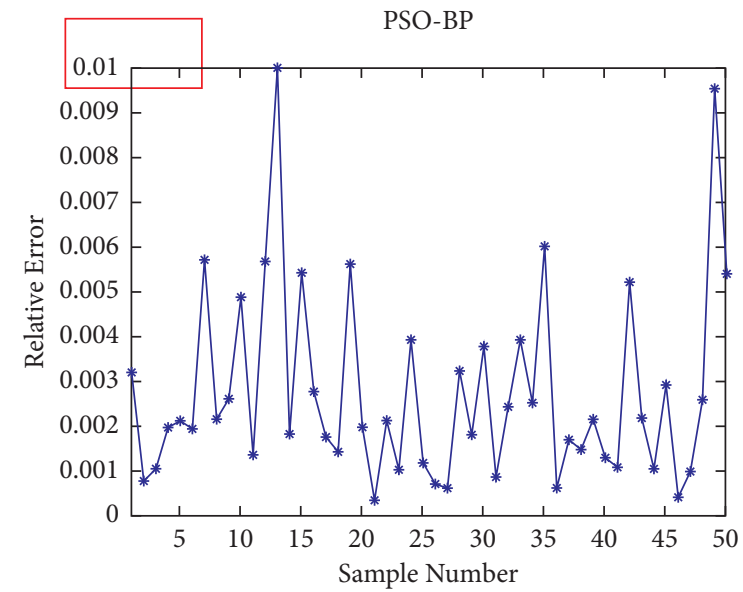

(b)

Figure 23: Continued. 


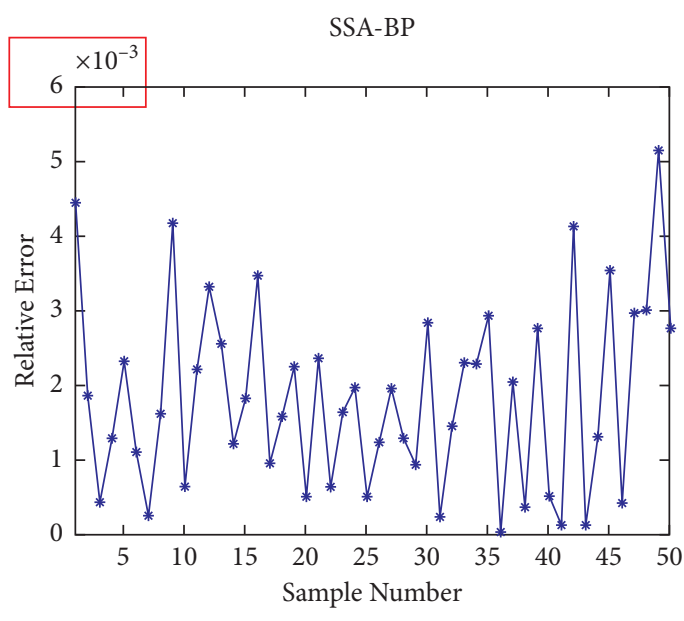

(c)

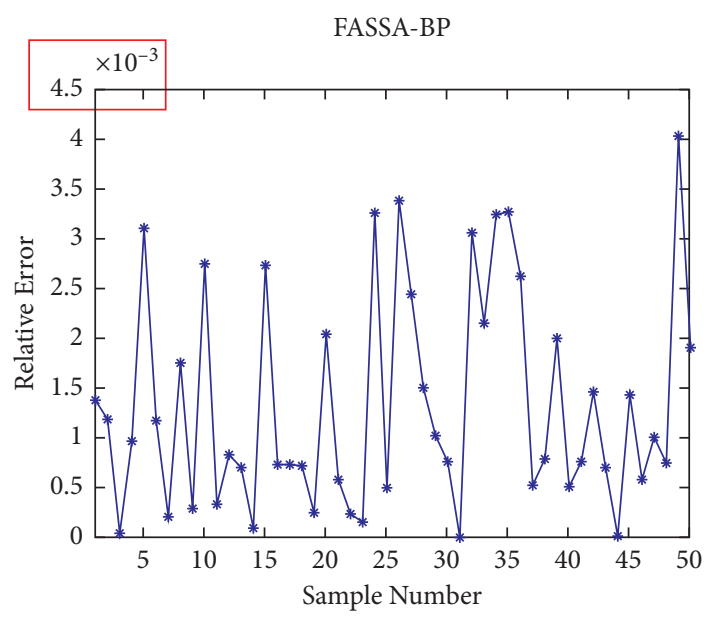

(d)

Figure 23: Relative error of each model test set sample in Experiment 2: (a) BP; (b) PSO-BP; (c) SSA-BP; (d) FASSA-BP.

Table 9: Experiment 2 data results of four model performance evaluation indexes.

\begin{tabular}{lcccc}
\hline Performance evaluation index & BP & PSO-BP & SSA-BP & FASSA-BP \\
\hline MRE (\%) & 0.4845 & 0.2722 & 0.1547 & 0.1441 \\
MSE (\%) & 8.4504 & 3.8626 & 1.9062 & 1.0151 \\
MAE (\%) & 25.39 & 14.63 & 0.11 & 7.93 \\
RMSE & 0.2957 & 0.2049 & 0.1329 & 0.1003 \\
R & 0.9824 & 0.9946 & 0.9997 & 0.9998 \\
\hline
\end{tabular}

TABLE 10: Relative error between the true value of the sample and the evaluation value of the FASSA-BP model.

\begin{tabular}{lcccccc}
\hline Test set samples & True value & Order of evaluation & Model evaluation value & Corresponding level & Relative error & Grade accurate level \\
\hline$S_{1}$ & 62 & Level three & 62.1728 & Level three & 0.0028 & Yes \\
$S_{2}$ & 55 & Level three & 55.0090 & Level three & 0.0002 & Yes \\
$S_{3}$ & 46 & Level two & 46.0456 & Level two & 0.0010 & Yes \\
$S_{4}$ & 53 & Level three & 52.9477 & Level three & 0.0010 & $\ldots$ \\
$\ldots$ & $\ldots$ & $\ldots$ & $\ldots$ & $\ldots$ & $\ldots$ & $\ldots$ \\
$\ldots$ & $\ldots$ & & $\ldots$ & $\ldots$ & $\ldots$ & $\ldots$ \\
$S_{47}$ & 62 & Level three & 62.0847 & Level three & 0.0014 & Yes \\
$S_{48}$ & 50 & Level two & 50.0713 & Level two & 0.0014 & Yes \\
$S_{49}$ & 43 & Level two & 42.9258 & Level two & 0.0017 & Yes \\
$S_{50}$ & 45 & Level two & 45.1327 & Level two & 0.0029 & Yes \\
\hline
\end{tabular}

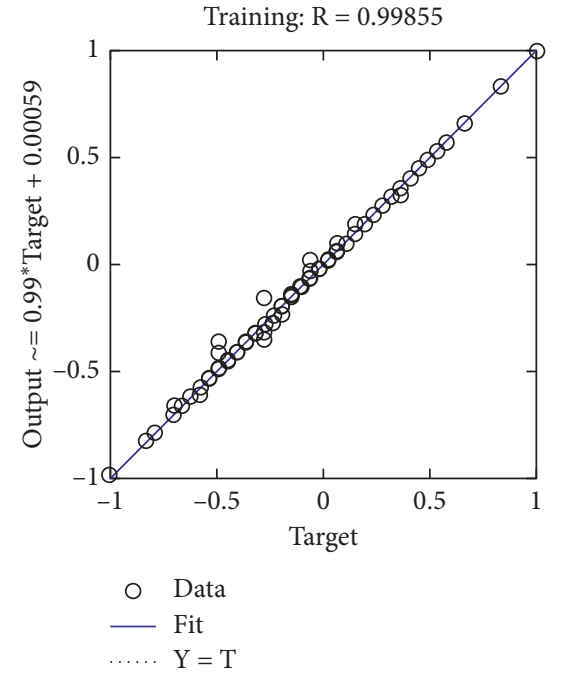

(a)

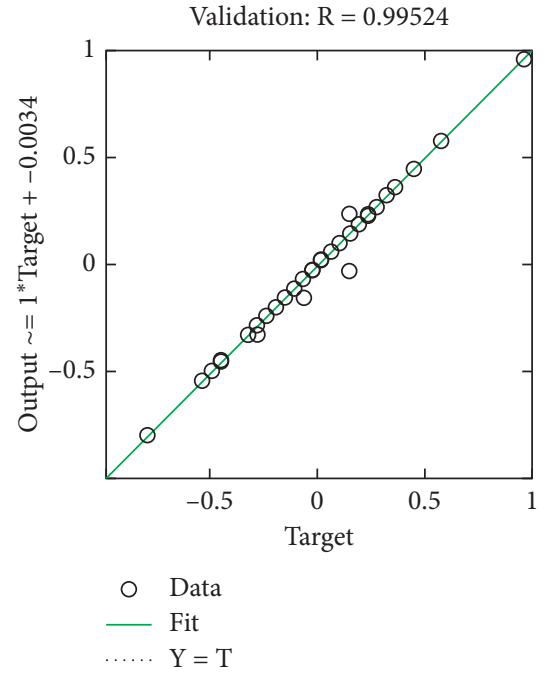

(b)

FIgURE 24: Continued. 


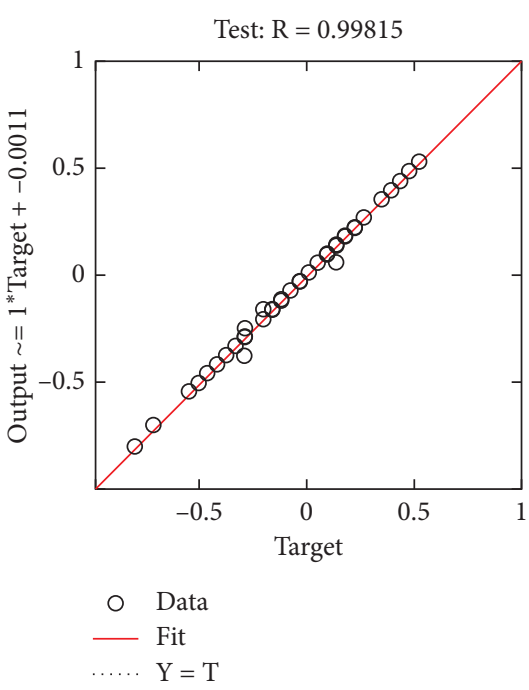

(c)

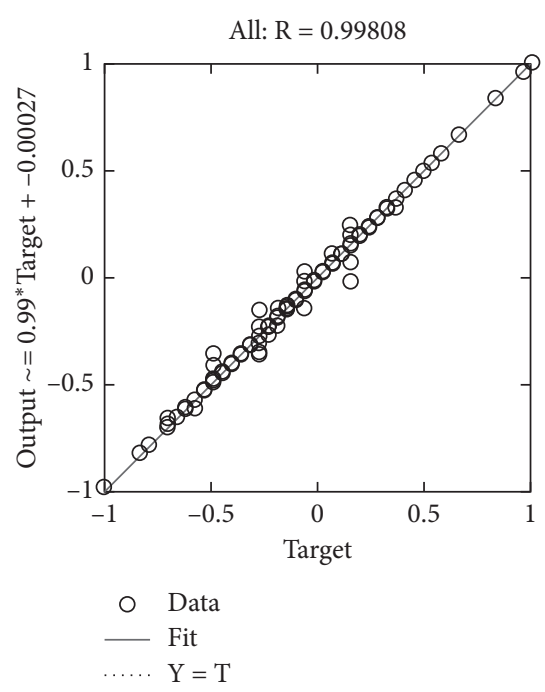

(d)

FIgURE 24: Regression analysis of the FASSA-BP model evaluation value and expected output value.

TABLE 11: Five intelligent manufacturing capability maturity levels of subsidiaries.

\begin{tabular}{lcr}
\hline Subsidiary number $C$ & Network output value $N$ & Corresponding grade $R$ \\
\hline$C 1$ & 59.0083 & Level three, $R 3$ \\
$C 2$ & 62.0154 & Level three, $R 3$ \\
$C 3$ & 54.9953 & Level three, $R 3$ \\
$C 4$ & 72.9602 & Level four, $R 4$ \\
$C 5$ & 43.0472 & Level two, $R 2$ \\
\hline
\end{tabular}

shortcomings, formulate reasonable improvement measures, and improve the level of enterprise intelligent manufacturing.

\section{Summary}

In order to help manufacturing enterprises diagnose the level of intelligent manufacturing capability and identify the gap, this paper conducts research on the maturity evaluation of intelligent manufacturing capability based on the maturity theory. By using the knowledge of computer science such as neural network and intelligent optimization algorithm, the evaluation model based on FASSA-BP algorithm is proposed. Improving and perfecting the theories and methods in the field of maturity evaluation of intelligent manufacturing capability can not only enrich the relevant theories of intelligent algorithms, but also broaden its application fields. The specific research work is summarized as follows:

(1) In view of the shortcoming that the sparrow search algorithm is easy to fall into local optimum, the disturbance strategy in the firefly algorithm is introduced to improve it, and an improved sparrow search algorithm (FASSA) is proposed. Low-dimensional and high-dimensional multimodal functions are selected to test the performance of the algorithm. Experiments show that, compared with SSA, FASSA has higher convergence accuracy and speed and better global search ability.

(2) An intelligent manufacturing capability maturity evaluation model based on FASSA-BP algorithm is established. Firstly, BP neural network is used to train and learn the relationship between the input sample data and the expected output value. Aiming at overcoming the defects of the initial weight and threshold randomization of BP neural network, we use FASSA to optimize the accuracy of network evaluation. Then, we compare and analyze the evaluation results of FASSA-BP model, BP model, SSA-BP model, and PSO-BP model from the aspects of accuracy and stability. The results show that the evaluation results of FASSA-BP model proposed in this paper are more accurate, which provides a new method for the evaluation of intelligent manufacturing capability maturity. Finally, it is applied to the actual case enterprises and combined with the evaluation results to give suggestions for improvement of intelligent manufacturing capabilities.

The research shows that the evaluation of the maturity of intelligent manufacturing capability by this model can help enterprises diagnose the problems existing in the construction of intelligent manufacturing and provide methods 
for enterprises to accurately improve their intelligent manufacturing capability. Although this method has certain practical significance and promotional value for enterprises to implement intelligent manufacturing, there is also the problem that the running time of FASSA-BP algorithm needs to be improved. It is necessary to further study this problem in the future.

\section{Data Availability}

The data used to support the findings of this study are included within the article.

\section{Conflicts of Interest}

The authors declare that there are no conflicts of interest regarding the publication of this paper.

\section{Acknowledgments}

This research was supported by the National Natural Science Foundation of China (Grant nos. 71801108, 62006091, and 62006092). The authors would like to thank Editage (http:// www.editage.cn) for their English language editing.

\section{References}

[1] G. Schuh, R. Anderl, and J. Gausemeier, Industrie 4.0 Maturity Index, National Academy of Science and Engineering, Munich, Germany, 2017.

[2] E. Gökalp, U. Şener, and P. E. Eren, "Development of an assessment model for industry 4.0: industry 4.0-MM," Communications in Computer and Information Science, vol. 99, pp. 128-142, 2017.

[3] B. Zhao, N. Guo, X. Yu et al., White Paper on the Maturity Model of Intelligent Manufacturing Capability, China Institute of Electronic Technology Standardization, Beijing, China, 2016.

[4] A. De Carolis, M. Macchi, E. Negri, and S. Terzi, "A maturity model for assessing the digital readiness of manufacturing companies," Advances in Production Management Systems. The Path to Intelligent, Collaborative and Sustainable Manufacturing, vol. 1, pp. 13-20, 2017.

[5] J. Hu and S. Gao, "Research and application of capability maturity model for Chinese intelligent manufacturing," Procedia CIRP, vol. 83, pp. 794-799, 2019.

[6] Y. Zhou, J. Zang, Z. Miao, and T. Minshall, "Upgrading pathways of intelligent manufacturing in China: transitioning across technological paradigms," Engineering, vol. 5, no. 4, pp. 691-701, 2019.

[7] W. Ren, H. Suo, and G. Zhao, "Smart factory capability maturity model in petrochemical industry," Computer and Applied Chemistry, vol. 36, no. 3, pp. 247-254, 2019.

[8] F. Simetinger and Z. J. Zhang, "Deriving secondary traits of industry 4.0: a comparative analysis of significant maturity models," Systems Research and Behavioral Science, vol. 37, no. 4, pp. 663-678, 2020.

[9] A. A. Wagire, R. Joshi, A. P. S. Rathore et al., "Development of maturity model for assessing the implementation of industry 4.0: learning from theory and practice," Production Planning \& Control, vol. 30, pp. 1-20, 2020.
[10] N. Ruiz, A. Giret, V. Botti, and V. Feria, "An intelligent simulation environment for manufacturing systems," Computers \& Industrial Engineering, vol. 76, pp. 148-168, 2014.

[11] M. Colli, O. Madsen, U. Berger, C. Møller, B. V. Wæhrens, and M. Bockholt, "Contextualizing the outcome of a maturity assessment for Industry 4.0," IFAC-PapersOnLine, vol. 51, no. 11, pp. 1347-1352, 2018.

[12] J. Lee, S. Jun, T.-W. Chang, and J. Park, "A smartness assessment framework for smart factories using analytic network process," Sustainability, vol. 9, no. 5, pp. 794-808, 2017.

[13] X. Liu, X. Zhou, B. Zhu, K. He, and P. Wang, "Measuring the maturity of carbon market in China: an entropy-based TOPSIS approach," Journal of Cleaner Production, vol. 229, pp. 94-103, 2019.

[14] J. Xiao, Z. Ying-qi, and J. Xu, "Research on intelligent manufacturing maturity evaluation model based on AHP and DHNN," Journal of Systems Science, vol. 28, no. 2, pp. 105$110,2020$.

[15] X. Xu and C. Xiao, "Research on evaluation system of intelligent manufacturing ability," Smart factory, vol. 6, pp. 59-62, 2018.

[16] Z. Zhi-qiang, "Research on evaluation of intelligent manufacturing capacity based on catastrophe progression method," Economic Forum, vol. 578, no. 9, pp. 27-32, 2018.

[17] K. Shao and Y. Wen, "Research on comprehensive evaluation of intelligent manufactuing capability bsed on factor Analysis," Logistics Sci-Tech, vol. 40, no. 7, pp. 116-120, 2017.

[18] S. Gillard, N. Banach, E. Barlow et al., "Developing and testing a principle-based fidelity index for peer support in mental health services," Social Psychiatry and Psychiatric Epidemiology, vol. 10, pp. 1-9, 2021.

[19] W. Yang, K. Xu, J. Lian, C. Ma, and L. Bin, "Integrated flood vulnerability assessment approach based on TOPSIS and Shannon entropy methods," Ecological Indicators, vol. 89, pp. 269-280, 2018.

[20] J. Xue and B. Shen, "A novel swarm intelligence optimization approach: sparrow search algorithm," Systems Science \& Control Engineering, vol. 8, no. 1, pp. 22-34, 2020.

[21] I. Coolen, L.-A. Giraldeau, and M. Lavoie, "Head position as an indicator of producer and scrounger tactics in a groundfeeding bird," Animal Behaviour, vol. 61, no. 5, pp. 895-903, 2001.

[22] Z. Barta, A. Liker, and F. Mónus, "The effects of predation risk on the use of social foraging tactics," Animal Behaviour, vol. 67, no. 2, pp. 301-308, 2004.

[23] W. D. Hamilton, "Geometry for the selfish herd," Journal of Theoretical Biology, vol. 31, no. 2, pp. 295-311, 1971.

[24] D. H. Wolpert and W. G. Macready, No Free Lunch Theorem for Search; Proceedings of the JPL, Santa Fe Institute, Santa Fe, NM, USA, 1995.

[25] X. Ding, M. Hasanipanah, H. N. Rad et al., "Predicting the blast-induced vibration velocity using a bagged support vector regression optimized with firefly algorithm," Engineering with Computers, vol. 10, pp. 1-12, 2020.

[26] C. Mei-ying, N. zhi-wei, and Z. Xu-hui, "Overview on glowworm swarm optimization or firefly alogorithm," Computer Science, vol. 42, no. 4, pp. 19-24, 2015.

[27] X. Ding, L. Shi, M. Li et al., "Research on intelligent manufacturing capability maturity evaluation based on BP neural network," Journal of Qingdao University (Natural Science Edition), vol. 32, no. 3, pp. 20-30, 2019.

[28] X. Xue, Y. Zhang, and K. Yu, "Research on the evaluation of intelligent manufacturing capability based on BP neural 
network," Journal of Software, vol. 39, no. 8, pp. 162-166, 2018.

[29] D. E. Rumelhart, G. E. Hinton, and R. J. Williams, "Learning representations by back-propagating errors," Nature, vol. 323, no. 6088, pp. 533-536, 1986.

[30] J.-c. Li, D.-L. Zhao, B.-F. Ge, K.-W. Yang, and Y.-W. Chen, "A link prediction method for heterogeneous networks based on BP neural network," Physica A: Statistical Mechanics and Its Applications, vol. 495, pp. 1-17, 2018.

[31] B. Liu, R. Wang, G. Zhao et al., "Prediction of rock mass parameters in the TBM tunnel based on BP neural network integrated simulated annealing algorithm," Tunnelling and Underground Space Technology, vol. 95, 2020.

[32] Y. Wu, R. Gao, and J. Yang, "Prediction of coal and gas outburst: a method based on the BP neural network optimized by GASA," Process Safety and Environmental Protection, vol. 133, pp. 64-72, 2020.

[33] J. Lyu and J. Zhang, "BP neural network prediction model for suicide attempt among Chinese rural residents," Journal of Affective Disorders, vol. 246, pp. 465-473, 2019.

[34] L. Xu, T. Quan, J. Wang, T. Aaron Gulliver, and K. N. Le, "GR and BP neural network-based performance prediction of dual-antenna mobile communication networks," Computer Networks, vol. 172, Article ID 107172, 2020.

[35] L. Wu, Y. Yang, and M. Maheshwari, "Strain prediction for critical positions of FPSO under different loading of stored oil using GAIFOA-BP neural network," Marine Structures, vol. 72, Article ID 102762, 2020

[36] B. Jia, R. Dong, and J. Du, "Ozone concentrations prediction in Lanzhou, China, using chaotic artificial neural network," Chemometrics and Intelligent Laboratory Systems, vol. 204, Article ID 104098, 2020.

[37] Y. Deng, H. Xiao, J. Xu, and H. Wang, "Prediction model of PSO-BP neural network on coliform amount in special food," Saudi Journal of Biological Sciences, vol. 26, no. 6, pp. 11541160, 2019.

[38] D. Ma, T. Zhou, J. Chen, S. Qi, M. Ali Shahzad, and Z. Xiao, "Supercritical water heat transfer coefficient prediction analysis based on BP neural network," Nuclear Engineering and Design, vol. 320, pp. 400-408, 2017.

[39] M. S. Muthuvalu, V. S. Asirvadam, and G. Mashadov, "Performance analysis of arithmetic mean method in determining peak junction temperature of semiconductor device," Ain Shams Engineering Journal, vol. 6, no. 4, pp. 1203-1210, 2015.

[40] K. Kraiwattanawong, N. Sano, and H. Tamon, "Investigation on porous properties of carbon/carbon composite cryogels by using weighted arithmetic mean," Microporous and Mesoporous Materials, vol. 231, pp. 57-65, 2016.

[41] L. Chen, X. Yang, C. Sun, Y. Wang, D. Xu, and C. Zhou, "Feed intake prediction model for group fish using the MEA-BP neural network in intensive aquaculture," Information Processing in Agriculture, vol. 7, no. 2, pp. 261-271, 2020. 